\title{
Fundamental beam-beam limit from head-on interaction in the Large Hadron Collider
}

\author{
Kazuhito Ohmi* \\ KEK, Oho, Tsukuba, 305-0801, Japan
}

Frank Zimmermann

CERN, 1211 Geneva 23, Switzerland

(Received 23 July 2014; revised manuscript received 13 April 2015; published 15 December 2015)

\begin{abstract}
The beam-beam limit at hadron colliders manifests itself in the form of degraded luminosity lifetime and/or reduced beam lifetime. In particular, for increasing beam intensity, the nonlinear beam-beam force causes incoherent emittance growth, while the (linear) coupling force between the two colliding beams can result in coherent beam-beam instabilities. These phenomena may be enhanced (or suppressed) by lattice errors, external noise, and other perturbations. We investigate the luminosity degradation caused both by incoherent emittance growth and by coherent beam-beam instability. The resulting beam-beam limit for an ideal machine and the of question how it is affected by some of the aforementioned errors are discussed in theory and simulation.
\end{abstract}

DOI: 10.1103/PhysRevSTAB.18.121003

PACS numbers: 29.20.D-, 29.20.db

\section{INTRODUCTION}

The beam-beam limit is conventionally characterized in terms of the permissible tune shift due to the beam-beam interaction, i.e., by a value for the maximum beam-beam tune shift. Most hadron colliders are designed so that the two counter-circulating beams collide at one or several interaction points with equal emittances $\left(\varepsilon_{x}=\varepsilon_{y}=\varepsilon\right)$ and equal beta functions $\left(\beta_{x}^{*}=\beta_{y}^{*}=\beta^{*}\right)$ in the horizontal and vertical plane. In this case and at zero crossing angle, for $p$ - $p$ collisions the beam-beam tune shift $(\xi)$ per interaction point is expressed by

$$
\xi=\Delta \nu=-\frac{N_{p} r_{p}}{4 \pi \gamma \varepsilon}
$$

where $\gamma, N_{p}$ and $r_{p}$ are the relativistic factor, bunch populations, and the proton classical radius. The total tune shift in one revolution, $\xi_{\text {tot }}$, is given by summing (1) over all interaction points.

Noticeable emittance growth occurs for collisions with a high tune shift. The beam-beam limit may be defined as the value of the tune shift above which the luminosity degradation due to the emittance growth is significant.

While the tune shift limit approaches $\xi \sim 0.1$ per interaction point for electron-positron colliders in the presence of strong synchrotron radiation damping [1], in

\footnotetext{
*Corresponding author. ohmi@ post.kek.jp

Published by the American Physical Society under the terms of the Creative Commons Attribution 3.0 License. Further distribution of this work must maintain attribution to the author(s) and the published article's title, journal citation, and DOI.
}

the past the beam-beam limit for hadron colliders had been supposed to be at least an order of magnitude lower than for lepton colliders, since the hadron beams, unlike the lepton beams, did not feature any damping mechanism for the transverse or longitudinal particle oscillations.

Based on the experience at the $\mathrm{S} p \bar{p} \mathrm{~S}$ collider [2], the Large Hadron Collider (LHC) was designed with a nominal beam-beam parameter of $\xi=0.0034$ for each of three interaction points. Many simulations using both strongstrong and weak-strong models were performed to predict the beam-beam limit in the LHC, e.g., Ref. [3]. These simulations indicated beam-beam parameters even much larger than 0.01 not to be associated with significant emittance growth per se. Therefore, if the real beam-beam tune shift of the LHC were actually limited around $\xi \sim 0.01$, other ingredients like noise or additional errors would need to be invoked to explain such a tune-shift limit.

Since the start of LHC beam operation, several beam experiments related to the beam-beam limit have been performed in the LHC. These demonstrated that beambeam tune shifts far larger than the design value could be reached without an appreciable degradation of the luminosity lifetime [4,5], which appears fully consistent with the past simulations.

In this article we explore the fundamental beam-beam limit for the LHC and we investigate various mechanisms potentially restricting the beam-beam performance. We will vary the bunch population to study the beam-beam limit as a function of the tune shift. After examining the limit for the ideal machine, we consider the additional effects of crossing angle, transverse offset, linear and chromatic optics errors at the interaction point, nonlinear fields in the interaction region, and external noise. Long-range parasitic beam-beam interactions are not considered in this article. 
Empirical data on the beam-beam limit is available from a few hadron colliders. A total beam-beam tune shift from two interaction points (IPs) of 0.03 was routinely achieved in the Tevatron [6], with a complex long-range collision scheme. The Tevatron was a single ring collider, where 36 bunches per beam encountered bunches of the other beam at a total of 70 locations in the arc. At the LHC, dedicated experiments have been performed, which, at least in the absence of parasitic encounters, reached a total beam-beam tune shift (sum of $\xi$ over two IPs) as high as 0.034 without any difficulty $[4,5]$. At the RHIC, the betatron tune is constrained to the range between the $2 / 3$ and $7 / 10$ resonances in order to preserve the proton polarization. For future operation at higher beam intensities the tune footprint shall still be confined to the same area in the tune plane with the help of electron lenses [7,8].

Understanding the beam-beam limit is crucial for the optimization of the future High Luminosity LHC (HLLHC), which may operate with about 3 times the brightness (bunch charge divided by transverse emittance) of the nominal LHC. Parameters of the LHC are summarized in Table I. Throughout this paper the number of interaction points is taken to be two. The design beam-beam parameter is 0.0034 per collision. In the actual LHC there are four interaction points (IPs), and a standard bunch collides at 2 IPs with low beta for highest luminosity $\left(\beta^{*}=0.55 \mathrm{~m}\right.$ at the ATLAS and CMS experiments) and at the other 2 IPs at a higher beta with lower luminosity $\left(\beta^{*}=3-10 \mathrm{~m}\right.$ for ALICE and LHCb). For the ALICE experiment in IP2 the colliding beams are also separated transversely by several times the rms beam size in order to further lower, and to control, the luminosity, which much reduces the contribution of this IP to the total beam-beam tune shift.

This paper is organized as follows. Section II summarizes the simulation method employed to study the beam-beam limit. The simulated beam-beam limit without errors, as inferred from both incoherent emittance growth and coherent instability, is discussed in Sec. III.
The beam-beam limit with optics errors and collision offset noise is discussed in Sec. IV. Section V draws some conclusions. The Appendixes A and B present numerical coefficients for the Hamiltonian amplitude-detuning and resonance-driving terms for a single beam-beam collision with horizontal crossing at the LHC, and analytical expressions for the emittance growth due to random offset noise in collision-based on a weak-strong model, respectively.

\section{SIMULATION METHOD}

Beam particles experience an electromagnetic force induced by the counter-rotating colliding beam. This force is strongly nonlinear as a function of the transverse amplitude, because the charge distribution of the beam is localized. The betatron amplitude of the beam particles may be subject to chaotic diffusion due to this nonlinear force, leading to emittance growth. This emittance growth is regarded as an incoherent phenomenon in which beam particles independently move in a given potential of the colliding beam. To study this type of emittance growth the weak-strong model is available, in which the weak and strong beams are represented by a group of particles being tracked and by a given (fixed) potential, respectively. The potential is taken to be the one of a Gaussian distribution for example.

Two colliding beams can move coherently, correlated with each other. A $\pi$ mode instability, in which the two beams oscillate with the betatron phase difference of $\pi$, is the typical phenomenon. The coherent beam-beam instability results in emittance growth due to the nonlinear interaction. For the study of the coherent beam-beam instability, the strong-strong model is used, where both beams are represented by (macro)particles, which interact with each other. The shape of each beam, which can deviate from a Gaussian as a result of the dynamical evolution, affects the beam-beam interaction. The motion of the particles in the two beams is treated self-consistently.

TABLE I. Parameters for LHC and HL-LHC. Two parameter sets are quoted for the HL-LHC, referring to the baseline bunch spacing of $25 \mathrm{~ns}$ and to a backup option of $50 \mathrm{~ns}$, respectively.

\begin{tabular}{|c|c|c|c|c|c|}
\hline & LHC(2013) & LHC(design) & HL-LHC(25 ns) & HL-LHC(50 ns) & Simulation model \\
\hline Circumference $(L[\mathrm{~m}])$ & \multicolumn{5}{|c|}{26,658} \\
\hline Energy $(E[\mathrm{TeV}])$ & 4 & 7 & 7 & 7 & 7 \\
\hline Normalized emittance $(\mu \mathrm{m})$ & 2.0 & 3.75 & 2.5 & 3.0 & 2.0 \\
\hline$\beta^{*}[\mathrm{~m}]$ & 0.6 & 0.55 & 0.15 & 0.15 & 0.55 \\
\hline rms bunch length $[\mathrm{m}]$ & \multicolumn{5}{|c|}{0.0755} \\
\hline Tune $(x / y / z)$ & & & $64.31 / 59.32 / 0$ & & \\
\hline Bunch population $N_{p}\left(10^{11}\right)$ & 1.65 & 1.15 & 2.2 & 3.5 & variable \\
\hline Number of bunches & 1380 & 2808 & 2808 & 1404 & $\cdots$ \\
\hline Bunch spacing [ns] & 50 & 25 & 25 & 50 & $\ldots$ \\
\hline Crossing angle $[\mu \mathrm{rad}]$ & 285 & 285 & \multicolumn{2}{|c|}{$0-590$} & $0-285$ \\
\hline Beam-beam parameter/IP & 0.009 & 0.0034 & $0.003-0.011$ & $0.005-0.014$ & variable \\
\hline Luminosity $\left(10^{34} \mathrm{~cm}^{-2} \mathrm{~s}^{-1}\right)$ & 0.7 & 1 & 5 (lev.) & 2.5 (lev.) & variable \\
\hline
\end{tabular}


For the beam-beam limit in hadron colliders, slow emittance growth and the associated lifetime degradation are the primary effects, as coherent dipolar instabilities can be suppressed by a bunch-by-bunch feedback system.

\section{A. Weak-strong model}

In the weak-strong model, particles in a (weak) beam move through another colliding (strong) beam, experiencing its electromagnetic field. The motion under the influence of this field is integrated along the bunch length $s$. A transfer map at the interaction point $s=0(+n L)$ is expressed by

$\boldsymbol{x}(0+\epsilon)=S \exp \left[-: \int_{-\Delta}^{\Delta} V_{0}^{-1}(s) H_{b b} V_{0}(s) d s:\right] \boldsymbol{x}(0-\epsilon)$,

where $S$ designates the $s$-ordered product and $\pm \Delta$ is the interaction region of the two beams, which is taken to be several times $\sigma_{z}$. [The $s$-ordered product is based on the same concept and definition as the $t$-ordered product commonly used.] $V_{0}$ denotes the transfer map in the drift space between slice-by-slice collision points,

$$
\begin{aligned}
V_{0}(s) & \equiv V_{0}(s, 0) \\
& =S \exp \left[-: \int_{0}^{s} H_{0} d s:\right] \\
& =\exp \left[-: \frac{p_{x}^{2}+p_{y}^{2}}{2} s:\right] .
\end{aligned}
$$

The operator :: represents the Poisson bracket, namely $: p_{x}: x=\left[p_{x}, x\right]=-1$. The integration in Eq. (2) is performed through discretization by slicing of the strong beam: that is, it is replaced by the product of the beambeam transfer map between the sliced beam at $z_{i}$ and a particle at $z$, with collision point $s_{i}=\left(z-z_{i}\right) / 2$. The drift transformation from $s=0$ to the collision point $s_{i}$ and its inverse in Eqs. (2) and (3) are repeatedly applied for the slice-by-slice collision $[9,10]$.

The colliding strong beam is assumed to be characterized by a round Gaussian distribution with the transverse position $\left[x_{i}(s), y_{i}(s)\right]$ and the rms size $\sigma_{r}(s)$. The transverse centroid position and the transverse size depend on $s$ due to the crossing angle and due to the hourglass effect, respectively. The beam size varies with the longitudinal position $s_{i}$ of the slice-by-slice collision point. Without crossing angle, the beam-beam transfer map $\left(e^{-: H_{b b}}: \boldsymbol{x}\right)$ for the round beam collision at $s_{i}$ is expressed by

$$
\begin{aligned}
& \Delta p_{r}\left(s_{i}\right)=\frac{2 N_{p, i} r_{p}}{\gamma} \frac{1}{r}\left[1-\exp \left(-\frac{r^{2}}{2 \sigma_{r}\left(s_{i}\right)^{2}}\right)\right] \\
& \Delta p_{z}\left(s_{i}\right)=\frac{N_{p, i} r_{p}}{\gamma} \frac{1}{\sigma_{r}\left(s_{i}\right)^{2}} \exp \left(-\frac{r^{2}}{2 \sigma_{r}\left(s_{i}\right)^{2}}\right) \frac{d \sigma_{r}^{2}\left(s_{i}\right)}{d z}
\end{aligned}
$$

where $N_{p, i}$ are the bunch population of the $i$ th beam slice and $r$ is the transverse distance, at $s_{i}$, between the centroid position of the $i$ th slice of the opposite beam and the particle being tracked, i.e., $r^{2}=\left(x\left(s_{i}\right)-x_{i}\left(s_{i}\right)\right)^{2}+\left(y\left(s_{i}\right)-y_{i}\left(s_{i}\right)\right)^{2}$. The same equations can be found in Refs. $[9,10]$. For a finite horizontal crossing angle, transformations $x^{*}=x+z \tan \left(\phi_{c} / 2\right), \quad \delta^{*}=\delta-p_{x} \tan \left(\phi_{c} / 2\right)$ and their inverse are executed before and after collision [11,12]. The distance is $x^{*}\left(s_{i}\right)-x_{i}^{*}\left(s_{i}\right) \approx x\left(s_{i}\right)+s_{i} \tan \phi_{c}$, where $s_{i}=\left(z-z_{i}\right) / 2$.

The bunch luminosity is calculated as

$$
\begin{aligned}
L / f_{\text {rev }}= & \sum_{i=1}^{n_{s l}} \frac{N_{p}}{n_{p}} \sum_{j=1}^{n_{p}} \frac{N_{p, i}}{2 \pi \sigma_{r}\left(s_{i j}\right)^{2}} \\
& \times \exp \left[-\frac{r\left(x_{j}\left(s_{i j}\right), y_{j}\left(s_{i j}\right)\right)^{2}}{2 \sigma_{r}\left(s_{i j}\right)^{2}}\right],
\end{aligned}
$$

where $N_{p, i}$ denotes the true number of beam particles in the $i$ th slice of the strong beam, $n_{p}$ the number of macroparticles in the weak beam, and $f_{\text {rev }}$ the revolution frequency. The weak beam is represented by $n_{p}=131$, 072 macroparticles. The luminosity in Eq. (5) is converted to the real bunch population $N_{P}\left[O\left(10^{11}\right)\right]$ of the weak beam through the factor $N_{p} / n_{p}$. The number of beam slices $n_{s l}$ is chosen as 10. For $\beta^{*} \gg \sigma_{z}$ and without a crossing angle a single slice would be sufficient. A number of $n_{s l}=5$ is sufficient for modeling crossing collisions with a Piwinski angle of about $\phi_{\text {piw }}=\phi_{c} \sigma_{z} /\left(2 \sigma_{x}\right) \approx 1$ [13].

\section{B. Strong-strong model}

The electromagnetic field induced by the colliding beams is calculated by solving the Poisson equation using the particle-in-cell method.

The beam-beam map of Eq. (2) is applied to both beams (first and second; $\boldsymbol{x}_{1}$ and $\boldsymbol{x}_{2}$ ), and $H_{b b}$ is expressed by

$$
H_{b b}=\phi_{2}\left(\boldsymbol{x}_{1}\right)+\phi_{1}\left(\boldsymbol{x}_{2}\right),
$$

where $\phi_{2,1}\left(\boldsymbol{x}_{1,2}\right)$ is the potential induced by the second and first beam at the position of the first or second beam, $\left(\boldsymbol{x}_{1,2}\right)$, respectively.

The potential $\phi$ is determined, using a particle-in-cell (PIC) approach, by the two-dimensional Poisson equation,

$$
\Delta_{\perp} \phi_{2 / 1}(x, y ; z, s)=\frac{r_{e}}{\gamma_{1 / 2}} \rho_{2 / 1}(x, y ; z, s)
$$

where $\Delta_{\perp}$ is the transverse Laplacian operator, $\rho$ denotes the particle density of the beam at a given location $(z, s)$, and $(x, y, z, s)$ are the coordinates of each tracked proton in its respective beam.

The three-dimensional effect (longitudinal force) is taken into account by $[13,14]$ 


$$
\Delta p_{z, 1,2}=-\frac{\partial \phi_{2,1}\left[s_{i}\left(z_{1,2}\right)\right]}{\partial z_{1,2}}
$$

For the case of a transversely Gaussian beam, this equation is the same as the second line of Eq. (4). We recall that the coordinate $z=(s-c t)$ can be interpreted as time, and $p_{z}=\left(p-p_{0}\right) / p_{0}=\left(E-E_{0}\right) / E_{0}$ (for ultrarelativistc particles) as the relative energy deviation. In the strong-strong simulation, Eq. (8) is calculated by differentiation with respect to time $t$, not with respect to any of the spatial coordinates.

The transformations of Eqs. (7) and (8) are repeated slice by slice using Eq. (2). The luminosity is calculated by summation over the overlap of the number of particles in the simulation cells as

$L / f_{\text {rep }}=\int \rho_{1}(x, y, z ; s) \rho_{2}\left(x, y, z^{\prime} ;-s\right) d x d y d z d z^{\prime}$,

where $s=\left(z-z^{\prime}\right) / 2$.

The three-dimensional strong-strong simulation is time consuming. Taking the number of slices equal to one, we obtain the simpler case of a two-dimensional simulation, which will be employed to study the coherent instability in Sec. III B.

\section{BEAM-BEAM LIMIT FOR IDEAL LHC}

\section{A. Incoherent emittance growth}

\section{Collision without crossing angle}

The incoherent emittance growth is studied using the weak-strong approach. We first consider a simple model consisting of the round beam collision from Eqs. (2)-(4) and a linear arc. This arc is approximated by a $6 \times 6$ matrix transformation $\left(M_{2 \times 2 \times 2}\right)$ of the form

$$
M_{2 \times 2 \times 2}=\left(\begin{array}{ccc}
M_{x} & 0 & 0 \\
0 & M_{y} & 0 \\
0 & 0 & M_{z}
\end{array}\right),
$$

where

$M_{i}(s)=\left(\begin{array}{cc}\cos \mu_{i}+\alpha_{i} \sin \mu_{i} & \beta_{i} \sin \mu_{i} \\ -\gamma_{i} \sin \mu_{i} & \cos \mu_{i}-\alpha_{i} \sin \mu_{i}\end{array}\right)$,

with $\mu_{i}=2 \pi \nu_{i}$ and $\mu_{z}<0$ for positive momentum compaction. The simulation is performed by tracking beam particles, alternately applying Eq. (4) to represent the collision, and the matrix transformation for motion through the arc. The beam parameters are given in Table I [15]. The simulation results strongly depend on the betatron tunes, the beam-beam parameter, and the Piwinski angle $\phi_{\text {piw }} \equiv \theta_{c} \sigma_{z} / 2 \sigma_{x}$. We vary the bunch population in order to study the beam-beam limit as a function of the tune shift.
A superperiodicity of 2 is assumed for the betatron phase between the 2 IPs. Breaking this superperiodicity almost always degrades the simulated performance.

The macroparticles are tracked over $10^{6}$ turns, and the luminosity is calculated as a turn-by-turn average over every 100 turns. Figure 1 shows the luminosity decrement for several beam-beam parameters obtained with this simple model. The plot (a) depicts the luminosity evolution for several values of $\xi_{\text {tot }}$. The luminosity decrements are estimated by fitting these evolutions. The plot (b) summarizes the luminosity decrement per turn as a function of the beam-beam parameter. The decrement $\Delta L / L_{0}=10^{-9}$ corresponds to a luminosity lifetime of about 1 day for the LHC, as its revolution frequency is roughly $10^{9}$ per day. The simulated beam-beam limit corresponds to a high total tune shift $\xi_{\text {tot }}>0.2$ at the working points $\left(\nu_{x}, \nu_{y}\right)=$ $(0.31,0.31)$ and $(0.31,0.32)$. Even for the injection tunes $(0.28,0.31)$, the limit is $\xi_{\text {tot }}>0.1$. For equal tunes, $(0.31,0.31)$, the beam-beam system has approximately one degree of freedom since $\beta^{*}>\sigma_{z}$, Therefore, the very high beam-beam limit $\xi>0.2$ is not surprising [16-18].

To analyze the mechanism of the luminosity degradation, a frequency map analysis (FMA) [19-21] is performed. A number of $40 \times 40=1600$ particles are initialized on an $x-y$ grid space with grid steps of $0.2 \sigma$, where $z=0$. Thereby, an $8 \sigma \times 8 \sigma$ area is covered in the transverse
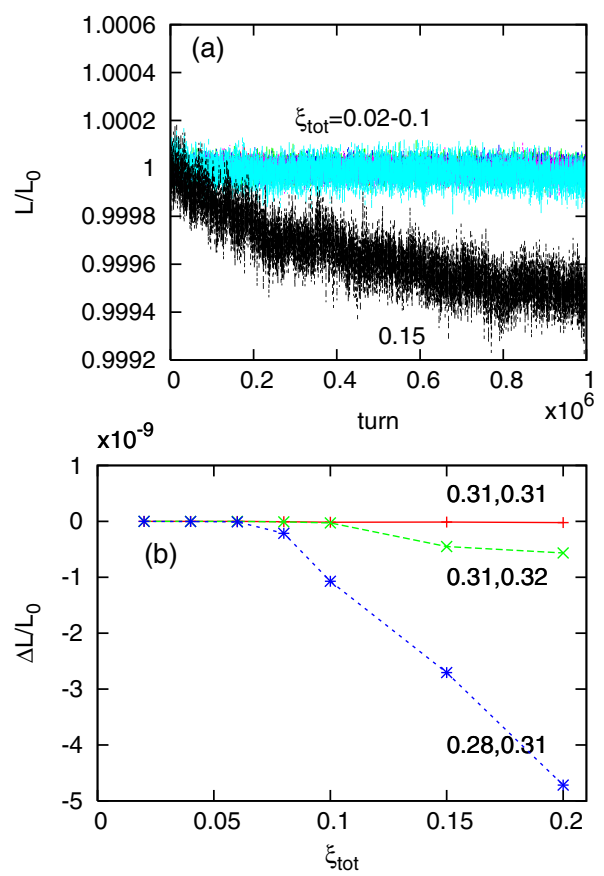

FIG. 1. Luminosity decrement obtained from the simple model consisting of the round beam interaction Eq. (4) and a linear arc. Picture (a) shows the luminosity evolution over $10^{6}$ turns for various values of $\xi_{\text {tot }}$ at $\left(\nu_{x}, \nu_{y}\right)=(0.31,0.32)$. Picture (b) summarizes the luminosity decrement per turn as a function of the total beam-beam parameter $\xi_{\text {tot }}$ at three different working points in the tune plane. 
amplitude space. The particles are tracked for 4096 turns. The tune is calculated from the turn-by-turn particle coordinates using a Hanning window over 1024 turns. This window is shifted every 128 turns. From the tune fluctuation a "diffusion index" is computed as [19]

$$
D=\frac{1}{2} \log _{10}\left(\delta \nu_{x}^{2}+\delta \nu_{y}^{2}\right) .
$$

Figure 2 shows the diffusion index obtained from the FMA. Pictures (a) and (b) depict this index in the tune and amplitude space, respectively, for $\xi_{\text {tot }}=0.06$. The size of the tune footprint displayed in the figure corresponds to $\xi_{\text {tot }}$. Several resonance lines of low or moderate order cross the areas of the tune footprint. The diffusion index remains small even near this resonance. If a resonance has a visible width the tune should fluctuate near its resonance line. This indicates that the resonances are not excited. Also in the amplitude space no sign of diffusion is seen.

The emittance growth is related to the local diffusion index and to the resonance width. The formation of resonance islands in phase space is a well-known phenomenon. The width in terms of transverse amplitude increases with the resonance strength and decreases for larger tune slope (i.e., tune shift with amplitude). Near the unstable fixed point of the resonance, the tune fluctuates leading to a local increase of the diffusion index, while the amplitude of a particle inside or close to a resonance may
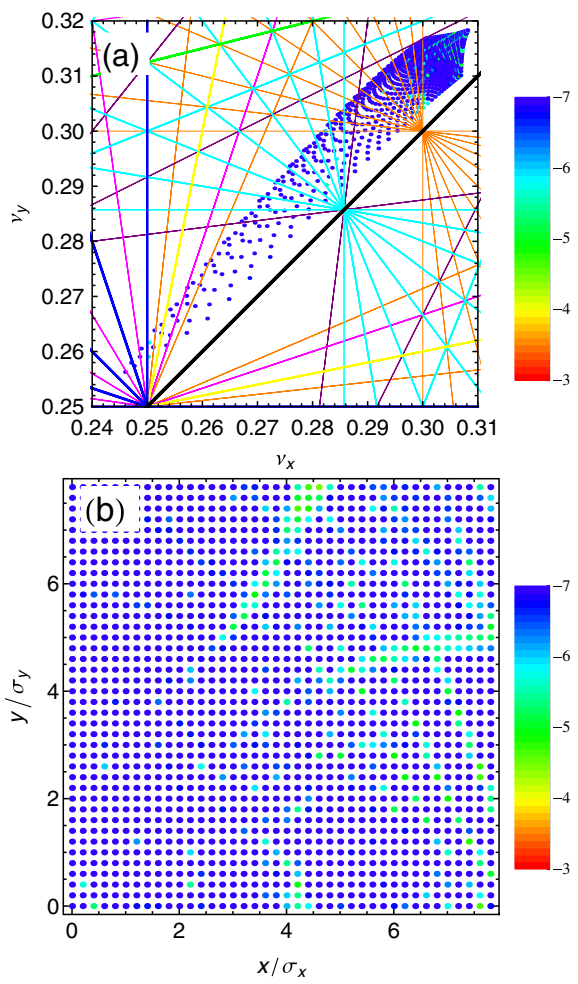

FIG. 2. Diffusion index in tune (a) and amplitude $(0-8 \sigma)$ (b) space for $\left(\nu_{x}, \nu_{y}\right)=(0.31,0.32)$ using the simple model. change over a range approximately equal to the resonance width. In the following we discuss emittance growth and luminosity degradation for various scenarios and assumptions.

\section{Collision with a crossing angle or a collision offset}

The crossing angle and collision offset degrade the luminosity performance, since the number of degrees of freedom of the beam-beam system is increased by the loss of collision symmetry: a crossing angle or a transverse offset break the symmetry between horizontal and vertical angles $p_{x}\left(p_{y}\right)$ or coordinates $x(y)$, respectively. In case of the collision with a crossing angle, the beam-beam interaction also depends on the longitudinal position $z$ of the particle, mainly through the coordinate $r$ in Eq. (4). By contrast, in case of a transverse collision offset, and no crossing angle, there hardly is any dependence on $z$ provided that $\sigma_{z}<\beta^{*}$.

Figures 3 and 4 present the simulated luminosity decrements for the crossing and horizontal offset collision, respectively. The half crossing angle is $\theta_{c} / 2=143 \mu \mathrm{rad}$, corresponding to a Piwinski angle $\phi_{\text {piw }} \equiv \theta_{c} \sigma_{z} / 2 \sigma_{x}=0.89$. LHC adopted horizontal/vertical crossing for two IPs. Tune operating point is $\left(\nu_{x}, \nu_{y}\right)=(0.31,0.32)$. Figure $3 \mathrm{com}-$ pares the luminosity decrement for horizontal/horizontal (blue) and horizontal/vertical crossing (cyan) with the case of zero crossing angle (red). For the alternating (horizontal/ vertical) crossing skew resonances are excited in addition to the normal resonances encountered for purely horizontal crossing. Nevertheless there is no significant difference in the simulated luminosity decrement: The alternating crossing yields somewhat better performance for the case of a crossing angle. Due to this crossing angle, the beam-beam tune shift in the crossing plane decreases to $63 \%$ of the value expected for a head-on collision, while the tune shift in the other plane is about $86 \%$ of the latter. Therefore, in the case of alternating crossing. the tune shift in either plane becomes $75 \%(=(86 \%+63 \%) / 2)$ of the head-on tune

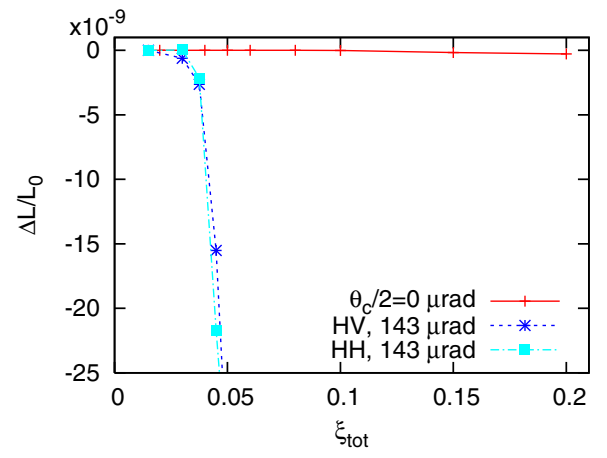

FIG. 3. Luminosity decrement as a function of total tune shift for collision with or without a crossing angle of $286 \mu \mathrm{rad}$, where the tune shift $\left(\xi_{\text {tot }}\right)$ is $75 \%$ of Eq. (1). Blue, cyan and red points refer to horizontal/vertical and horizontal/horizontal crossings or to no crossing angle, respectively. 


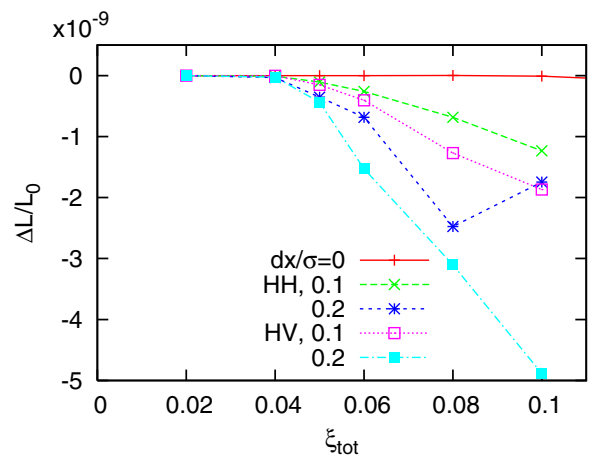

FIG. 4. Luminosity decrement as a function of total beam-beam tune shift for collisions with a horizontal offset equal to $0,10 \%$ and $20 \%$ of the rms beam size, and zero crossing angle. The abbreviations "HH" and "HV" refer to horizontal/horizontal and horizontal/vertical offsets, respectively.

shift (see Eq. (1)). The nonzero crossing angle also decreases the geometrical luminosity to $75 \%$ of the head-on case.

The geometrical luminosity reduction due to a collision offset of $\Delta x=0.2 \sigma$ is only $1-2 \%$, and roughly the nominal tune shift is experienced for a collision with such an offset. Further below the tune shift for both cases is studied using the FMA analysis.

In the case of the crossing-angle collision for $\xi_{\text {tot }} \geq$ 0.038 the luminosity degrades by more than $\Delta L / L_{0}>$ $10^{-9}$ per turn, as is illustrated in Fig. 3. The luminosity degradation for the offset collision is mild compared with the one for the crossing-angle collision. The beam-beam limit, determined from the condition $\Delta L / L_{0}=10^{-9}$, corresponds to a total tune shift of 0.038 for the collision at the nominal LHC crossing angle, and to $\xi=0.06-0.08$ for the offset collision with $\Delta x=0.1 \sigma_{x}$.

A frequency-map analysis (FMA) sheds some light on the degradation mechanism for collisions with either a crossing angle or transverse offset. Figures 5 and 6 present the FMA results for alternating crossing planes and for an alternating (hor./vert.) transverse offset of $0.2 \sigma_{x y}$, respectively. The bunch population is the same for Fig. 2, resulting, for Fig. 5, in a total tune shift (with nonzero crossing angle) of $\xi_{\text {tot }}=0.06 \times 0.75=0.045$. For both the crossing-angle and offset collisions, resonances of order 7 , 10 and 13 are seen in the FMA. The order of a resonance $m_{x} \nu_{x}+m_{y} \nu_{y}=n$ with $m_{x}$ and $m_{y}$ integer is given by the sume of the moduli $\left|m_{x}\right|+\left|m_{y}\right|$. All 7-th order resonances (i.e., $\left(m_{x}, m_{y}\right)$ with $\left|m_{x}\right|+\left|m_{y}\right|=7$ ) should be excited for the alternating (horiz./vert.) crossing and offset, but only resonances of even number $m_{y}$ for horizontal-horizontal crossing angles. The tune points are scattered near the resonance lines. Some red points are seen in the vicinity of the $(7,0)$ line. The corresponding red points are also seen at around $x / \sigma_{x} \approx 2, y / \sigma_{y} \approx 0$ in the amplitude planes of Figs. 5(b) and 6(b). The resonance lines with positive
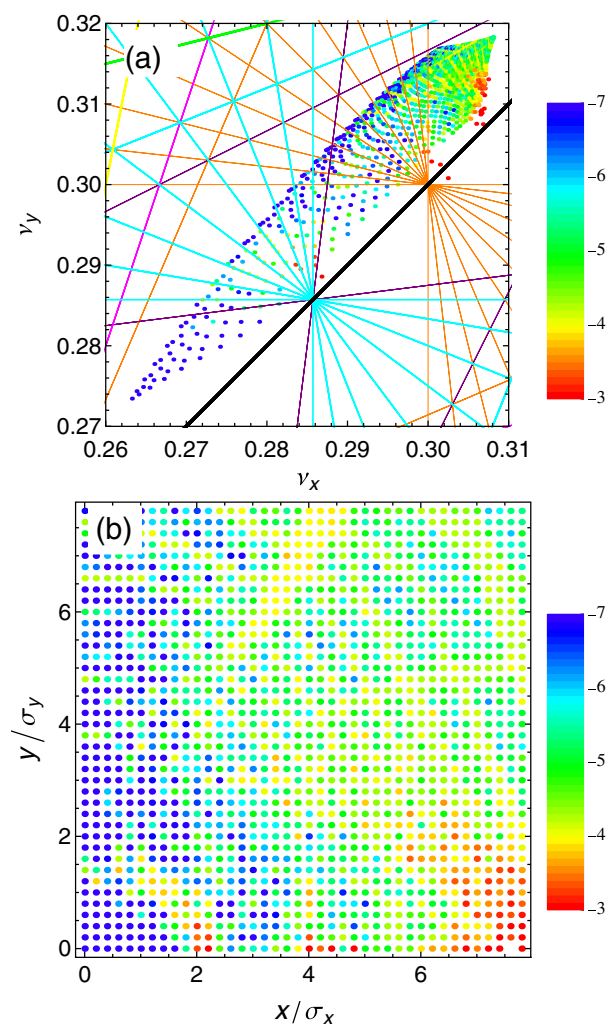

FIG. 5. Diffusion index for the crossing-angle collision with alternating horizontal and vertical crossing in two IPs. The top and bottom pictures show tune and amplitude spaces.
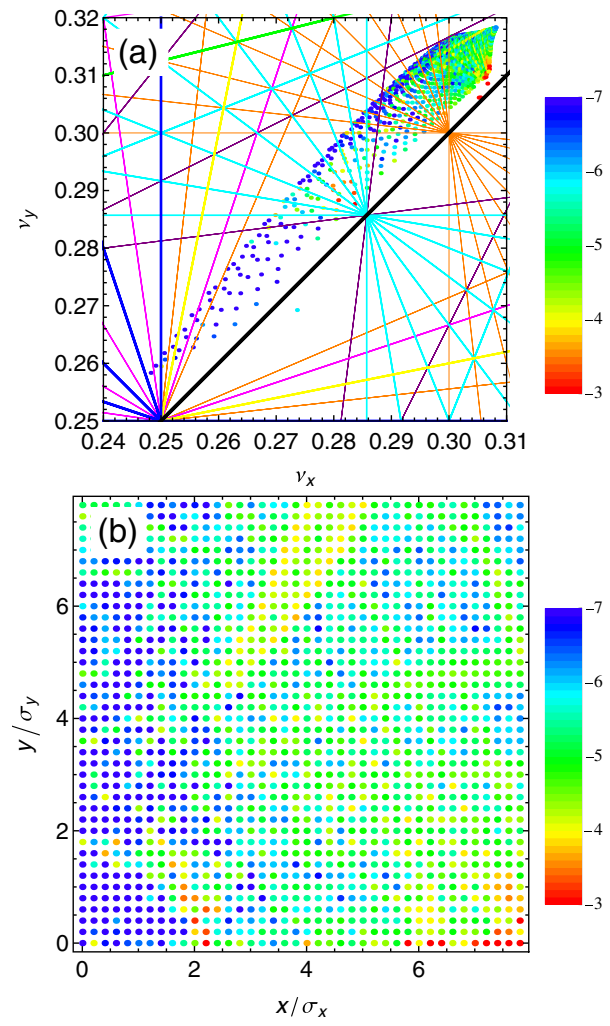

FIG. 6. Diffusion index for collision with a transverse offset of $20 \% \sigma$. The top and bottom pictures show tune and amplitude spaces. 

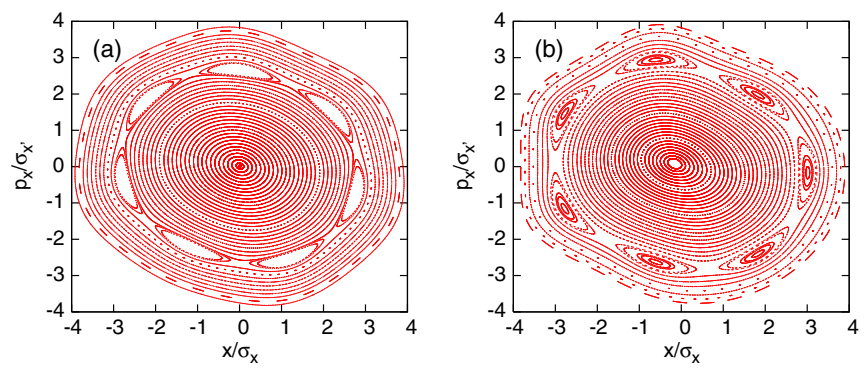

FIG. 7. Phase space $\left(x-p_{x}\right)$ plot for the crossing-angle (a) and offset collision (b).

slope may be of order $11(4,-7)$ and/or $13(5,-8)$. The 10-th, 12-th and 13-th order resonances visible at large amplitudes $\left(x / \sigma_{x} \approx 4-7\right)$ do not seem to contribute to the luminosity degradation. The size of the tune footprint of Fig. 5 agrees with the expected tune shift for the crossingangle collision, since $2 \times 0.03 \times 0.75 \approx 0.045$.

Figure 7 shows simulated trajectories in the $x-p_{x}$ phase space for a single collision with (a) horizontal crossing angle and (b) horizontal offset, at zero vertical amplitude $(y=0)$. In both pictures 7 islands, corresponding to the $(7,0)$ resonance, are seen. While the diffusion index and the resonance width are similar for the collision with crossing angle and with transverse offset, the emittance growth rate for the case of nonzero crossing angle is stronger than the one with a transverse offset collision, as can be seen by comparing Figs. 3 and 4.

The island amplitudes at $2.5-3 \sigma_{x}$ in Fig. 7 seem to be somewhat different from the amplitudes of highest diffusion index, around $2 \sigma_{x}$, in Figs. 5 and 6. One reason for the difference lies in the detailed footprint areas for horizontal/ horizontal (HH) and horizontal/vertical (HV) crossing [Figs. 5 and 6]. For HH crossing the tune shifts are $\xi_{x, \text { tot }}=$ $0.06 \times 0.63=0.0378$ and $\xi_{y, \text { tot }}=0.06 \times 0.86=0.0516$, which is to be compared with $\xi_{x, \text { tot }}=\xi_{y, \text { tot }}=0.06 \times 0.75=$ 0.045 for the HV crossing.

\section{Tayler map analysis and resonance width}

We study the transverse resonance in more detail for the case of a single collision with horizontal crossing. The resonances for the collision with crossing angle can be investigated by a Taylor map analysis. The one-turn Taylor map containing the beam-beam interaction is calculated up to 12-th order for the betatron variables. The map is factorized as [22]

$$
M \exp (-: H:)
$$

where $M$ represents the linear part of the map, which consists of the arc transfer map and the linear beam-beam tune shift, and $H$ the nonlinear part in the form of polynomials higher than 2 nd order. $H$ can be expressed through action-angle variables, $J_{i}$ and $\phi_{i}, i=x, y$.
The Fourier decomposition into harmonics of the betatron phases $\phi_{i}$ yields

$H=H_{00}\left(J_{x}, J_{y}\right)+\sum G_{m_{x}, m_{y}}\left(J_{x}, J_{y}\right) \exp \left(m_{x} \phi_{x}+m_{y} \phi_{y}\right)$.

The terms $H_{00}$ and $G_{m_{x}, m_{y}}$ characterize the amplitude dependent tune shift and the resonance strength, respectively. The coefficients of $H_{00}$ and $G$ can be calculated by the code $\mathrm{SAD}^{+}$, as discussed in Appendix A. The beambeam force (Eq. (4)) is strongly nonlinear, so that it is difficult to express as a Tayler expansion. Nevertheless, an expansion up to 12-th order, approximately valid for $\sqrt{2 J_{x, y} / \varepsilon_{x y}}<2.5$ in case of a Gaussian beam, can be employed to analyze the emittance growth occurring near the beam core. The difference between the 12-th order expansion and the exact expression of Eq. (4) increases from $2 \%$ to $75 \%$ for $x$ amplitudes between 2 and $2.5 \sigma_{x}$.

A resonance is represented by a curve in action space $\left(J_{x}, J_{y}\right)$ satisfying the relation

$m_{x}\left(\nu_{x, 0}+\left.\frac{\partial H_{00}}{\partial J_{x}}\right|_{J=J_{R}}\right)+m_{y}\left(\nu_{x, 0}+\left.\frac{\partial H_{00}}{\partial J_{y}}\right|_{J=J_{R}}\right)=n$.

where $J=J_{R}$ means $J_{x}=J_{x, R}$ and $J_{y}=J_{y, R}$. The resonance half width is expressed by

$$
\Delta J_{x}=2 m_{x} \sqrt{\frac{G_{m_{x}, m_{y}}\left(J_{x, R}, J_{y, R}\right)}{\Lambda}},
$$

where

$\Lambda=m_{x}^{2} \frac{\partial^{2} H_{00}}{\partial J_{x}^{2}}+m_{x} m_{y} \frac{\partial^{2} H_{00}}{\partial J_{x} \partial J_{y}}+\left.m_{y}^{2} \frac{\partial^{2} H_{00}}{\partial J_{y}^{2}}\right|_{J=J_{R}}$.

Figure 8 presents the resonance lines and their widths in the action space, considering the case of horizontal/horizontal crossing. Overall the resonance locations and widths are consistent with the diffusive areas of Figure 5. For example, the width of the $(7,0)$ resonance is $\pm \Delta J_{x}=$ $4.6 \times 10^{-11} \mathrm{~m}$ for $J_{x}=6.0 \times 10^{-10}$ and $J_{y}=0 \mathrm{~m}$. In phase space, the resonance island is located at $x / \sigma_{x}=$ $\sqrt{2 J_{x} / \varepsilon_{x}}=2.1$ and its width is $\Delta x / \sigma_{x}= \pm 0.1$. The island position and width agree fairly well with those in Figure 7.

One difference should, however, be pointed out between the purely horizontal crossing model analyzed here and the case of horizontal/vertical crossing considered in Fig. 5. Namely, in Fig. 8 the tune footprint $\left(\xi_{x, \text { tot }}, \xi_{y, \text { tot }}\right)=$ $(0.0378,0.0516)$ overlaps with the linear coupling resonance, where several resonance lines intersect at $(0.286$, 0.286). This resonance intersection is evident in Fig. 8. 


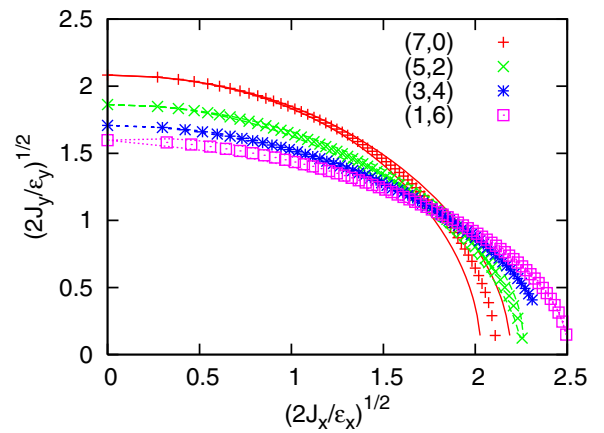

FIG. 8. Resonance lines and their widths in the amplitude space given by Taylor map analysis for a horizontal/horizontal crossing. The units on the horizontal and vertical axes are equivalent to $x / \sigma_{x}$ and $y / \sigma_{y}$.

By contrast, the tune footprint shown in Fig. 5(a) does not cross the linear coupling resonance, and, as a consequence, for this example of horizontal/vertical crossing the resonance lines do not intersect in amplitude space. Interestingly and importantly, the differences in the emittance growth obtained for horizontal/horizontal and verti$\mathrm{cal} /$ horizontal crossing are not significant, as can be seen in Figs. 3 and 4. Therefore, the large resonance width induced by the crossing angle appears to be the key ingredient responsible for the simulated emittance growth, whereas the crossing scheme chosen for multiple interaction points is of minor relevance. In particular, crossing the linear resonance at $(0.286,0.286)$, as in the case of horizontal/ horizontal crossing, does not seem to enhance the emittance growth. Indeed, the linear resonance $\left(\nu_{x}=\nu_{y}\right)$ has little impact on the emittance growth for a round beam, because the sum of the two transverse action variables, $J_{x}+J_{y}$, is conserved on such a resonance.

\section{Synchrotron motion}

We now discuss why the crossing-angle collision is far more harmful than the offset collision. An essential element is that the beam-beam force strongly depends on $z$ for the crossing-angle collision, while only faintly for the offset collision.

The luminosity evolution of the crossing-angle and offset collision is shown in Fig. 9 as red and green lines, respectively. The one for the crossing collision without synchrotron motion is plotted as a blue line. The luminosity decrement for the crossing collision-angle without synchrotron motion (blue) shows a behavior similar to the one for the offset collision (green). The decrement changes dramatically when synchrotron motion is included (the red curve).

The FMA analysis shown in Fig. 5 was performed for $z=0$. The horizontal crossing angle induces additional resonances for particles at $z=0$, which arise due to the asymmetry with respect to $p_{x}$. The characteristics of the transverse resonances for this case is similar to the one for

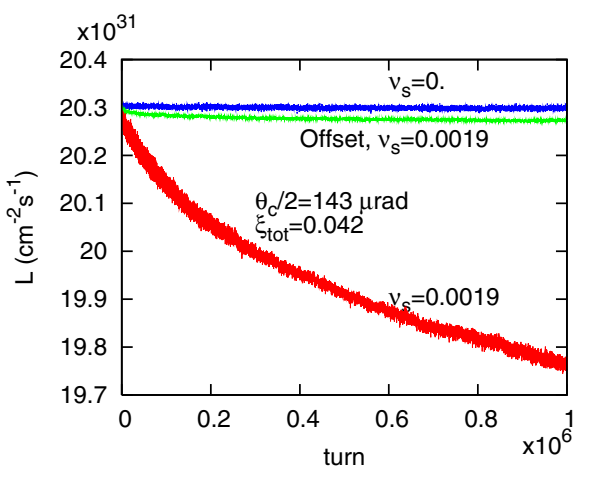

FIG. 9. Luminosity evolution for the arc transformation with or without synchrotron oscillation.

the offset collision. Namely, in the (horizontal) crossing collision, particles with a finite value of $z$ collide with the other beam at a transverse offset $\delta x=z \theta_{c} / 2$, and the effective horizontal rms size of the beam collision becomes $\sqrt{\sigma_{x}^{2}+\left(\theta_{c} \sigma_{z} / 2\right)^{2}}$. A collision offset induces the same resonances. However, in the case of the crossing angle synchrotron motion results in a slow variation of the resonance strength and resonance location, i.e., in a variation of the resonance amplitude $\left(J_{R}\right)$ and width $(\Delta J)$, experienced by individual particles. Therefore, during a synchrotron period (or half period), these particles can be trapped, moved, and released by the resonances. This process, which repeats every synchrotron period, causes an enhancement of the emittance growth $[18,23]$. The same diffusion mechanism appears if the dispersion at the collision point or the chromaticity are nonzero, as we will illustrate later.

Figure 10 presents some FMA results for $x-z$ motion in the case of horizontal/horizontal crossing. The diffusive area in the horizontal plane increases for finite synchrotron oscillation amplitudes, $z \leq 0.5 \sigma_{z}$. In particular, the 7-th order resonance becomes stronger at the synchrotron amplitudes. Emittance growth can be caused by a resonance overlap between synchrotron side bands. We remark that the synchrotron sideband $\nu_{x} \pm \nu_{s}\left(\nu_{s}=0.0019\right)$ is not visible with the resolution of this picture, and that the longitudinal oscillations, i.e., synchrotron tune or synchrotron amplitude, are not noticeably affected by the transverse motion, since $\varepsilon_{z} \gg \varepsilon_{x y}$. The emittance growth is also explained by the afore-mentioned resonance crossing. Though it is not clear whether these two understandings mention the same physics, as far as this FMA results, understanding with the resonance crossing seems to be feasible.

\section{Tune scans}

The beam-beam limit depends on the operating point in tune space. The luminosity degradation is caused by resonances as was discussed above. Therefore, it has long been a common understanding that the beam-beam limit is 

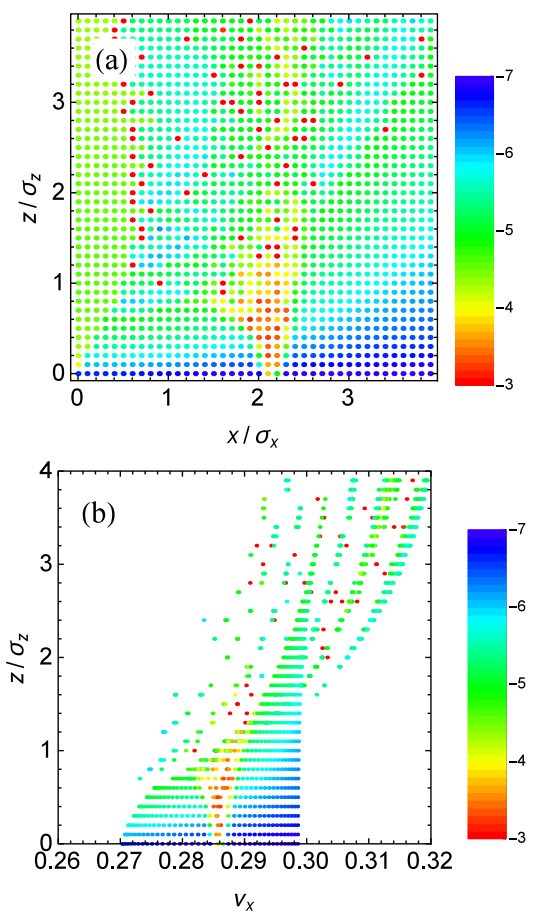

FIG. 10. Diffusion index for the crossing-angle collision with horizontal crossing only, and zero vertical amplitude $(y=0)$. The $x$ and $z$ amplitudes are scanned from 0 to $4 \sigma$ with a $0.1 \sigma$ step.

determined by the combination of the beam-beam tune shift and the distance in tune from strong resonances.

Figure 11 shows the luminosity decrement as a function of tune for collisions with the crossing angle $\theta_{c} / 2=$ $143 \mu \mathrm{rad}$ in the horizontal and vertical planes, for a beam-beam tune shift of $\xi=0.045$. The two transverse tunes are varied together, while keeping the difference of the horizontal and vertical tunes constant, equal to $\nu_{x}-\nu_{y}=0,-0.01,-0.02,-0.04$, and -0.06 . The lines for $0,-0.01$, and -0.04 are shown in the figure. The luminosity decreases monotonically as the tune difference increases. the tune space around the diagonal is mostly free of resonances (see Sec. III).

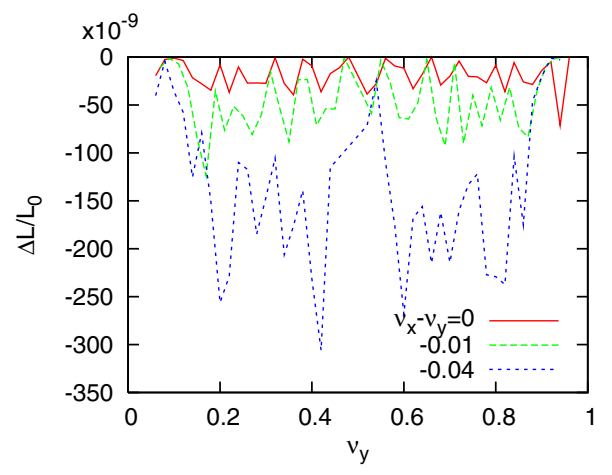

FIG. 11. Luminosity decrement as a function of tune for $\theta_{c} / 2=143 \mu \mathrm{rad}$. The three curves correspond to $\nu_{x}-\nu_{y}=0$, -0.01 , and -0.04 . The total beam-beam tune shift is $\xi_{\text {tot }}=0.045$.
In actual beam operation arranging for equal tunes or at least close tunes in the two planes is a delicate manoeuvre. Small perturbations can lead to exchanges between the $x$ and $y$ modes. A small difference, e.g., 0.01, allows for more stable operational conditions, the LHC design working point $\left(\nu_{x}, \nu_{y}\right)=(0.31,0.32)$ being one of the best choices. The optimum working points found in our simulations are slightly below the half integer or the integer resonance, e.g., $(0.47,0.48)$ and $(0.97,0.98)$.

The tune scans for collisions with $\theta_{c}=0$ yield much better results. The decrement is smaller than $10^{-9}$ per turn for most working points in the regions $0.12<\nu_{x}=\nu_{y}<0.5$ and also at $0.12<\nu_{x}<0.5$ at $\nu_{y}=0.32$.

We suppose that the luminosity degradation for different operating points can be explained by the resonance-driven diffusion mechanism discussed earlier in this section.

\section{B. Coherent beam-beam instability}

The onset of coherent instability is studied by the strong-strong simulation (using the simulation code BBSS [13,24]). The simulation is performed using a two-dimensional model. Thus the crossing angle and longitudinal effects are not taken into account. In the case of KEKB, any transverse coherent motion was suppressed in three dimensional simulations [13]. Based on the KEKB experience the results from the two-dimensional model should, therefore, be considered pessimistic. However, the synchro-betatron modes are missed by the two dimensional model. In the simulations for the LHC no coherent motion is observed at the nominal operating point. The appearance of the coherent beam-beam instability strongly depends on the tunes, because the instability is driven either by linear beambeam resonances or by mode coupling between higherorder beam-beam modes. Keeping the horizontal tune constant, we scan the vertical tune to look for instabilities. Figure 12 shows the luminosity decrement as a function of the vertical tune, as simulated with the two dimensional model $(x-y)$. A luminosity degradation is seen near the integer and half-integer resonances as well as at $\nu_{y} \approx 59.36$. At the same time the coherent $\pi$ mode instability is seen near the integer tune, in the range $\nu_{y}=59.0-59.2$. Since the number of interaction points is two, the corresponding phase advance between successive interaction points is $\sim 29.5-29.6$ in units of $2 \pi$. This instability is predicted by linear theory [25].

A coherent instability is also observed near the half integer tune for $\nu_{y}=59.52-59.54$, which corresponds to a phase advance between interaction points of 29.7629.77. In addition, near the half integer tune a coherent variation of the beam size is seen, related to the quadrupole mode, which is absent near the integer resonance.

Finally, the instability at $\nu_{y} \sim 59.36$ is enhanced by a transverse collision offset (see the second picture of Fig. 12). Frequency spectra near $\nu_{y} \sim 0.36$ are shown in 

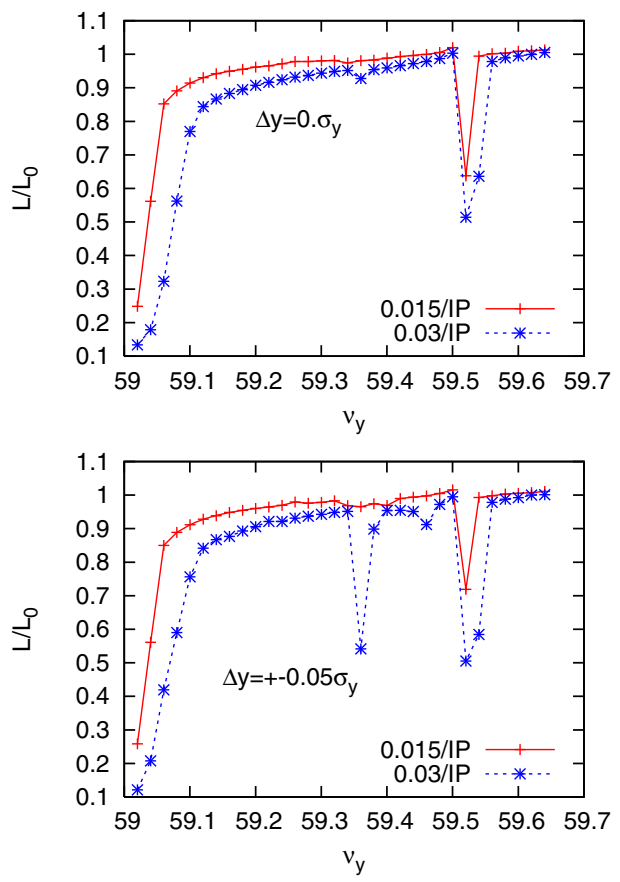

FIG. 12. Luminosity degradation as a function of vertical tune for different values of the beam-beam tune shift per IP (colors) without (top) and with a transverse offset of $0.05 \sigma$ (bottom).

Fig. 13. This coherent instability, which is predicted by theory [26], is caused by mode coupling between the dipole $\pi$ mode and the quadrupole mode. This interpretation is confirmed by comparing the Fourier transforms of the beam position and beam size. A broad peak of quadrupole mode at $1-2 \nu_{y}$, which is seen in the FFT of the beam size, shifts towards lower frequencies for increasing tune. Two peaks corresponding to the $\pi$ and $\sigma$ modes are visible at $\nu_{y}=0.34$. Though the $\sigma$ and quadrupole modes overlap here, the beam remains stable. On the other hand, the overlap of the $\pi$ and quadrupole modes at $\nu_{y}=0.36-0.38$
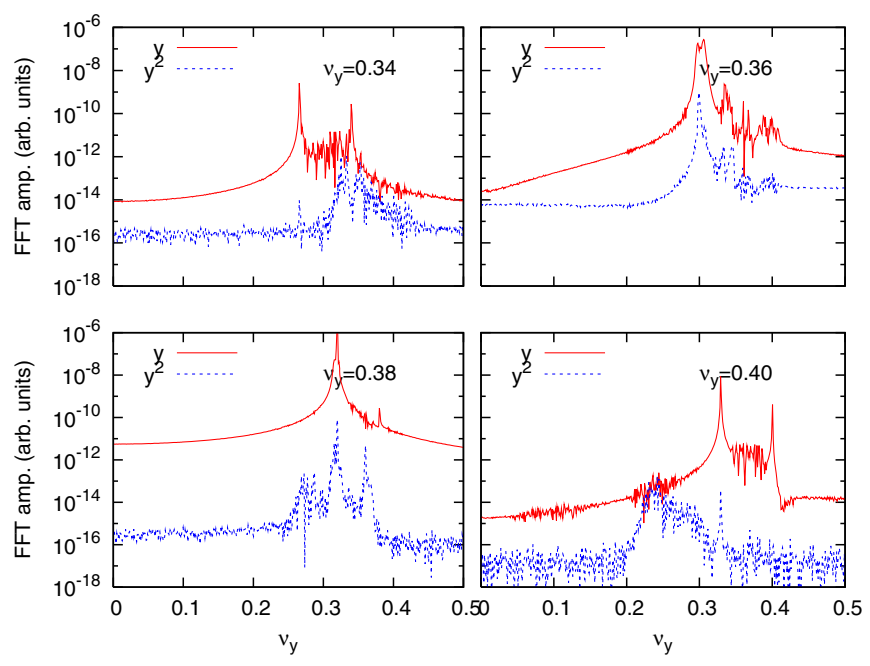

FIG. 13. Frequency spectra for $\langle y\rangle$ and $\left\langle y^{2}\right\rangle$. The four plots depict the spectra for tunes, $0.34,0.36,0.38$ and 0.40 . results in a coherent instability, which can indeed be explained by the coupling of these two modes.

These simulation results indicate that the coherent instability is not the source of the beam-beam limit for hadron colliders, since it is easily suppressed by a proper choice of tune.

\section{BEAM-BEAM LIMIT FOR LHC WITH ERRORS}

In the real machine, the beam-beam performance is likely to be influenced, and limited, by errors. In our beam-beam map, only the optics parameters at the interaction point affect the beam-beam collision, while the effect of the ring is characterized through global accelerator parameters, such as emittance, tune, amplitude-dependent tune shift, etc. In $\mathrm{e}^{+} \mathrm{e}^{-}$ colliders, the optimization of the optics parameters at the IP, especially for $x-y$ coupling, vertical dispersion and local chromatic dependence, proved to be essential for achieving the highest luminosity $[27,28]$. In hadron colliders, collision with round beams is popular. Under these conditions the effects of $x-y$ coupling or chromatic $x-y$ coupling should be different from those in $\mathrm{e}^{+} \mathrm{e}^{-}$colliders.

In a strongly nonlinear system, a fluctuation of the dynamical variables enhances the emittance growth. Therefore, a fluctuation of the collision offset can degrade the luminosity performance of a collider. In $\mathrm{e}^{+} \mathrm{e}^{-}$colliders, an intrinsic fluctuation is caused by the synchrotron radiation: the magnitude of this fluctuation for a single particle is given by $\delta x / \sigma_{x}=\sqrt{2 / \tau_{x}}$ where $\tau_{x}$ denotes the horizontal emittance damping time due to the synchrotron radiation in units of turns. An additional fluctuation of the collision offset smaller than the one caused by the synchrotron radiation does not affect the beam-beam limit. However, in the case of hadron colliders, the damping time $\tau$ is extremely long, e.g., $\tau \approx 10^{9}$ turns ( $\sim 1$ day) in the LHC. This implies that in hadron colliders the sensitivity to external fluctuations could be much enhanced compared with the case of $\mathrm{e}^{+} \mathrm{e}^{-}$colliders.

In this section we discuss the LHC tolerances to both optics errors and collision fluctuations.

\section{A. $x-y$ coupling and dispersion}

The $r$ parameters which characterize the $x-y$ coupling are introduced by factorizing the revolution matrix at the collision point as

$$
M_{4}=R^{-1} M_{2 \times 2} R
$$

where

$$
R=\left(\begin{array}{cccc}
r_{0} & 0 & -r_{4} & r_{2} \\
0 & r_{0} & r_{3} & -r_{1} \\
r_{1} & r_{2} & r_{0} & 0 \\
r_{3} & r_{4} & 0 & r_{0}
\end{array}\right)
$$




$$
M_{2 \times 2}=\left(\begin{array}{cc}
M_{x} & 0 \\
0 & M_{y}
\end{array}\right)
$$

and

$$
M_{i}(s)=B^{-1}(s) U B(s),
$$

with

$$
U=\left(\begin{array}{cc}
\cos \mu_{i} & \sin \mu_{i} \\
-\sin \mu_{i} & \cos \mu_{i}
\end{array}\right), \quad B=\left(\begin{array}{cc}
\frac{1}{\sqrt{\beta_{i}}} & 0 \\
-\frac{\alpha_{i}}{\sqrt{\beta_{i}}} & \sqrt{\beta_{i}}
\end{array}\right)
$$

with $i=x$ or $y$.

The $r_{i}$ 's characterize the tilt angles in phase space at the IP for the elliptic trajectories of each betatron mode, e.g., $r_{1} \approx-\langle x y\rangle /\left\langle x^{2}\right\rangle$ is the tilt angle in the $x-y$ space for the horizontal (dominant) betatron mode if $\alpha_{i}=0$ at IP. Concerning the other $r_{i}{ }^{\prime}$ s, $r_{2} \approx-\left\langle p_{x} y\right\rangle /\left\langle p_{x}^{2}\right\rangle, r_{3} \approx$ $-\left\langle x p_{y}\right\rangle /\left\langle x^{2}\right\rangle$, and $r_{4} \approx-\left\langle p_{x} p_{y}\right\rangle /\left\langle p_{x}^{2}\right\rangle$ are the tilt angles in the respective coordinate-momentum space. The diagonal component of $R$ is $r_{0}=\sqrt{1-r_{1} r_{4}+r_{2} r_{3}}$.

Figure 14 presents the simulated luminosity degradation due to residual $x-y$ coupling and spurious dispersion at the interaction point.
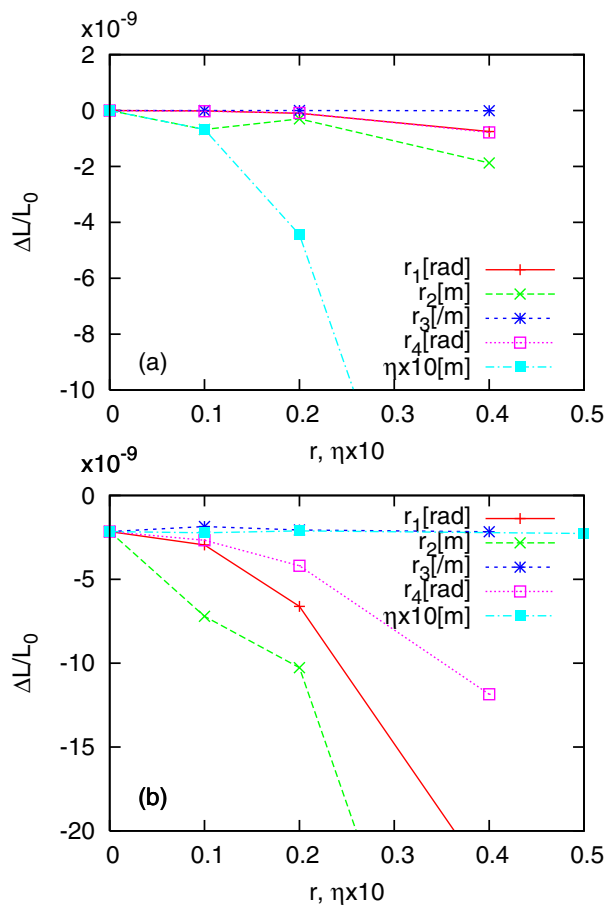

FIG. 14. Luminosity decrement as a function of residual $x-y$ coupling and spurious dispersion, for the cases without $\left[\xi_{\text {tot }}=0.06\right]$ (top) and with crossing angle $\left[\theta_{c}=295 \mu \mathrm{rad}\right.$, $\left.\xi_{\text {tot }}=0.038\right]$ (bottom). The units are $r_{2}(\mathrm{~m})$ and $r_{3}\left(\mathrm{~m}^{-1}\right)$, and dimensionless for $r_{1}$ and $r_{4}$.
The tolerance depends on the beam-beam parameter. From Fig. 14, for $\xi_{\text {tot }}=0.06$ without crossing angle we infer a tolerance of $r_{i} \sim 0.3-0.4$ and $\eta \sim 0.01 \mathrm{~m}$, while for the crossing-angle collision with $\xi_{\text {tot }}=0.038$ we have $r_{i} \sim 0.1$.

The beam-beam force of Eq. (4) is a function of $r=\sqrt{x^{2}+y^{2}}$. In the presence of coupling, $r$ in the force is replaced by $r\left(R^{-1} \boldsymbol{x}\right)$. Using the matrix form of Eq. (19), we have

$r^{2}=x^{2}+y^{2}+2\left(r_{1}-r_{4}\right) x y+2 r_{2}\left(x p_{y}+p_{x} y\right)$,

where only the first-order terms of the coupling are taken into account. The beam-beam force is independent of $r_{3}$. The effect of $r_{1}$ and $r_{4}$ is similar, since the force is a function of $r_{1}-r_{4}$. For $r_{2}$ in standard units the sensitivity is $1 / \beta \sim 2 \mathrm{~m}^{-1}$ stronger than for $r_{1}, r_{4}$, because $x, y \sim \beta p_{x, y}$.

It is then no surprise that the luminosity degradation is significant for the crossing-angle collision, since here, in Eq. (22), the term $x$ is replaced by $x-\theta_{c} z$. The force containing $z$ leads to a worse luminosity performance as we have seen in the aforementioned simulation results.

For a similar reason a clear luminosity degradation is also seen in the case of nonzero IP dispersion for the collisions without a crossing angle. The underlying dynamical mechanism is the same as the one for the crossing angle. With nonzero IP dispersion, a particle collides with a transverse orbit offset which depends on the energy deviation, and varies from turn to turn due to the particle's synchrotron motion. This modulated offset induces resonances. The size of the offset is $\eta \sigma_{\delta} / \sigma_{x}=0.09$ for $\eta=0.01 \mathrm{~m}$, considering the LHC rms momentum spread of $\sigma_{\delta}=1.13 \times 10^{-4}\left(\delta=\Delta p / p_{0}\right)$. The normalized offset is still small compared with $\theta_{c} \sigma_{z} / \sigma_{x}=0.89$, which explains why no clear luminosity degradation due to the additional dispersion is seen in the case of the crossingangle collision. For the latter, the degradation due to the crossing angle is dominant and, in addition, the beam-beam parameter is small.

The results of Fig. 14 are plausible from these discussions.

\section{B. Chromaticity and IR nonlinearity}

Chromaticity in tunes and beta functions at IP affects the luminosity degradation [29]. The chromaticity is modeled by an effective Hamiltonian (generating function) of the form [30],

$$
H_{x, \text { chrom }}=\left(a_{x} x^{2}+2 b_{x} x p_{x}+c_{x} p_{x}^{2}\right) \delta,
$$

where $a_{x}, b_{x}, c_{x}$ are related to $d \nu_{x} / d \delta, d \beta_{x} / d \delta$, and $d \alpha_{x} / d \delta$. The same description can be applied for the vertical motion. Figure 15 presents the simulated luminosity decrement as a function of chromaticity $\left(d \nu_{x} / d \delta=d \nu_{y} / d \delta\right)$ without and with a crossing angle, keeping the chromatic aberrations for 
$\alpha_{x y}$ and $\beta_{x y}$ equal to zero. The luminosity degradation is significant for the crossing-angle collision.

For $d \nu / d \delta \sim 10$ and $\sigma_{\delta}=1.13 \times 10^{-4}$, the tune variation due to chromaticity is one order of magnitude lower than the beam-beam tune shift. However, the tune variation with the synchrotron period results in a periodic variation of the resonant amplitude $J_{R}$, for the resonances existing within the area of the tune spread. The chromaticity, thereby, causes an effect similar to those of the crossing angle and of the IP dispersion. The enhancement of the emittance growth for the crossing-angle collision seen in Fig. 15 appears reasonable.

In this figure, the cyan line shows an interesting phenomenon for the combined effect of beam offset (without crossing angle) and chromaticity. The collision with transverse offset only was nondetrimental and resulted in a small luminosity decrement even in the presence of resonances (Fig. 4). Adding to the transverse offset the tune variation due to chromaticity and synchrotron motion results in a larger luminosity decrement.

Figure 16 presents the luminosity decrement as a function of the chromatic beta aberration at the IP (assuming $d \beta_{x} / d \delta=d \beta_{y} / d \delta$ ), where now the tune chromaticity is set to zero $(d \nu / d \delta=0)$. Also here the simulated luminosity strongly degrades for the cases with crossing angle. The responsible mechanism is again similar. The beam-beam tune shift depends on the beta function, which varies due to the synchrotron motion. A beta variation is around $10 \%$ for
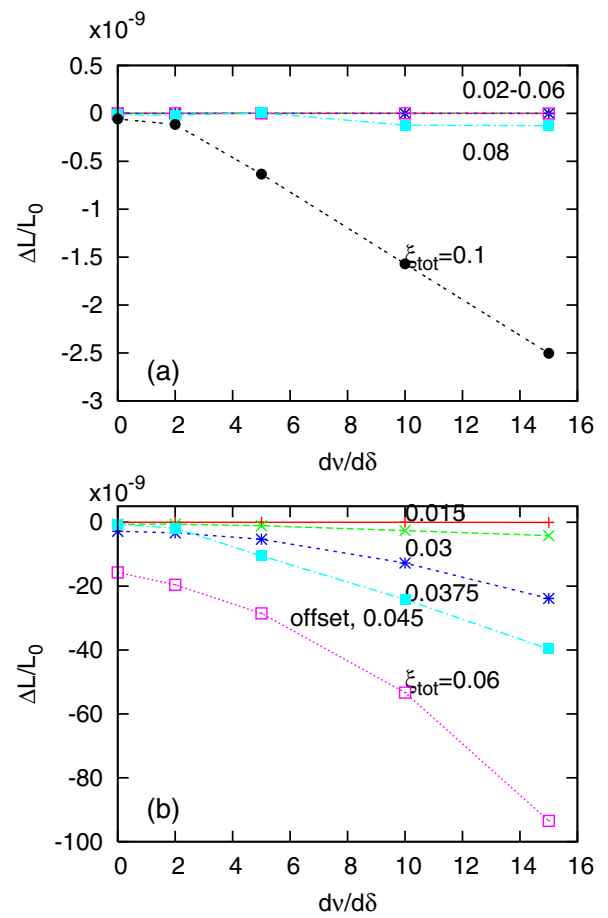

FIG. 15. Luminosity decrement as a function of chromaticity without (a) and with crossing angle (b). The cyan line in (b) refers to a transverse offset of $0.2 \sigma$ and total tune shift $\xi_{\text {tot }}=0.045$.
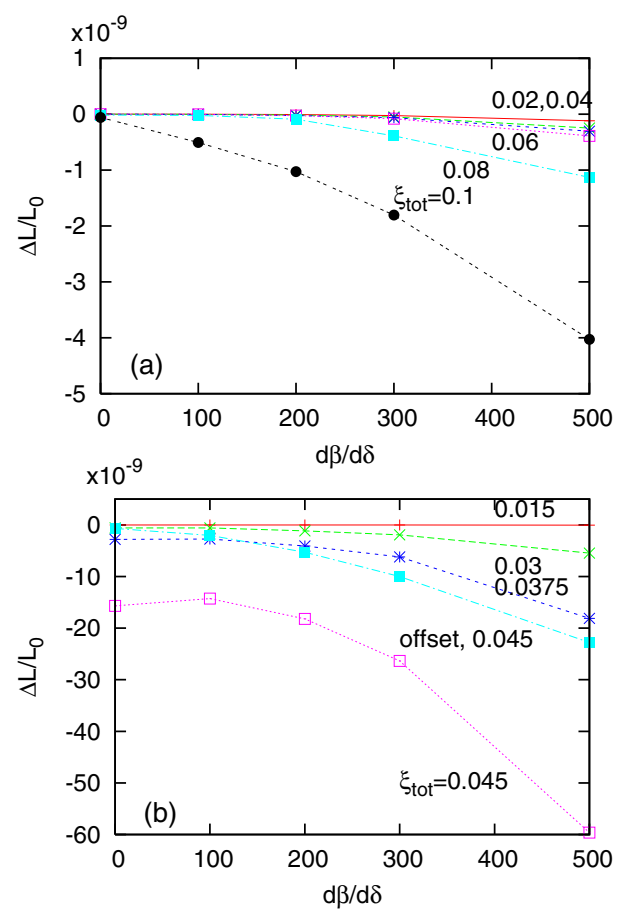

FIG. 16. Luminosity decrement as a function of chromatic beta aberration at IP without (a) and with crossing angle (b). The cyan line in (b) refers to a transverse offset of $0.2 \sigma$ and total tune shift $\xi_{\text {tot }}=0.045$.

$d \beta / d \delta=100 \mathrm{~m}$ and results in a tune shift variation of about $10 \%$. The chromatic beta $d \beta / d \delta \sim 100$ causes an effect comparable to the effect of the chromaticity $d \nu / d \delta$. The combined effect of beta variation and a transverse beam offset of $0.2 \sigma$ (for zero crossing angle) is also seen in Fig. 16.

The final triplet in the interaction region is the dominant source of nonlinearity for the squeezed accelerator lattice. The quadrupole magnets contain nonlinear field components and are located in a region with extremely high beta functions. For this reason, in collision the dynamic aperture is mainly limited by the nonlinearity of the IR magnets. The beam-beam interaction, coupled to this IR nonlinearity, may limit the luminosity performance. We employ a simple IR model [31] to study the resulting beam-beam limit. The description of the nonlinearity is obtained from magnetic field measurements of IR quadrupole magnets [32]. In our model the linear chromaticity induced in the IR is compensated by using the effective Hamiltonian of Eq. (23). The uncorrected nonlinear chromaticities are $d^{2} \nu / d \delta^{2}=$ 362 and $d^{2} \beta / d \delta^{2}=19.6$. In the simulation this nonlinearity degrades the luminosity only at high beam-beam parameter, $\xi>0.1$. For beam-beam tune shifts $\xi<0.1$, the effect of the IR nonlinearity is not significant. Therefore, we do not present these results here in greater detail.

In the real machine, resonances excited by nonlinear magnetic fields (lattice sextupoles, octupoles, field errors, 
etc.) also affect the emittance growth. The aforementioned IR model may be insufficient for a proper evaluation of these resonances driven by the real accelerator lattice with imperfections. In general, the important question is whether the relevant resonances excited by the nonlinear magnetic fields lie within the tune footprint area of the beam-beam force, whether their widths are sufficiently large [33], and if their widths and resonant amplitudes $J_{R}$ vary with the synchrotron motion. As we have shown, resonances are more detrimental in the case of the crossing-angle collision, and chromaticity enhances the emittance growth.

Lattice nonlinearities may affect, and ultimately, limit the beam-beam performance of the Large Hadron Collider as the $\beta^{*}$ will further be reduced, e.g., to values a factor 2-4 below the original design. A detailed study of this complex phenomenon for the LHC, and for its HL-LHC upgrade, is beyond the scope of the present paper and planned for the future.

\section{Random noise for collision offset}

We now discuss the interplay of the beam-beam effect with fast external noise, which introduces a fluctuation of the beam trajectory. Adding a fast offset fluctuation with turn-by-turn or longer-term correlation to the nonlinear beam-beam interaction causes emittance growth and luminosity degradation. Phase noise of crab cavities as well as the detection (position monitor) and kicker noise of the bunch by bunch feedback system, or magnetic-field variation due to power-converter ripple, are possible sources of such fluctuation.

We first use the weak-strong model to explore the beambeam effects in the presence of a fluctuation. In our model, an external noise is introduced for the transverse position of the strong beam at the collision point.

We consider an orbit (horizontal position) shift of the strong beam of the form,

$$
\Delta x_{i+1}=(1-1 / \tau) \Delta x_{i}+\delta x \hat{r},
$$

where $\Delta x_{i}$ is the horizontal orbit shift on the $i$ th turn. $\tau, \delta x$, and $\hat{r}$ are a (normalized) damping time, a constant characterizing the random fluctuation amplitude, and a Gaussian random number with unit standard deviation, respectively. This equation is known as the OrnsteinUhlenbeck process. In the following we will refer to this type of noise as the first type. A vertical noise without correlation to the horizontal one can be considered independently.

All particles in the weak beam experience the fluctuation of the strong beam. Thereby, a transverse collective motion is induced. This collective motion results in emittance growth through filamentation due to the nonlinear beambeam force.
For $\tau \gg 1$ the stable (equilibrium) amplitude of the fluctuation of the strong beam is given by

$$
\Delta x^{2}=\left\langle\Delta x_{n \rightarrow \infty}^{2}\right\rangle=\frac{\tau \delta x^{2}}{2} .
$$

The correlation function between the $i$ th and $i+n$th turns is expressed through the damping time as

$$
\left\langle\Delta x_{\ell} \Delta x_{\ell+n}\right\rangle=\Delta x^{2} e^{-|n| / \tau},
$$

i.e., the damping time $\tau$ can be identified with the correlation time of the fluctuation. For turn-by-turn white noise, which corresponds to $\tau=1$, the correlation function is expressed by

$$
\left\langle\Delta x_{\ell} \Delta x_{\ell+n}\right\rangle=\Delta x^{2} \delta_{n 0},
$$

where $\delta_{n 0}$ designates the Kronecker delta.

The beam oscillates at the betatron frequency. This motivates considering another (second) type of noise of the following form:

$$
\begin{aligned}
\Delta x_{i+1} & =(1-1 / \tau)\left(\Delta x_{i} \cos \mu_{o}+\Delta p_{i} \sin \mu_{o}\right)+\delta x \hat{r} \\
\Delta p_{i+1} & =(1-1 / \tau)\left(-\Delta x_{i} \sin \mu_{o}+\Delta p_{i} \cos \mu_{o}\right)+\delta x \hat{r},
\end{aligned}
$$

where $x$ and $p$ are the coordinate and its canonical momentum normalized by the beta function, so that $J=\left(x^{2}+p^{2}\right) / 2$, and $\mu_{o}=2 \pi \nu_{o}$ is the betatron tune multiplied by $2 \pi$. The stable dipole oscillation amplitude is expressed by an equation analogous to Eq. (25). The correlation function contains the betatron tune as follows:

$$
\left\langle\Delta x_{\ell} \Delta x_{\ell+n}\right\rangle=\Delta x^{2} e^{-|n| / \tau} \cos n \mu_{o} .
$$

For turn-by-turn white noise, the diffusion of $J$ is expressed by

$$
\begin{aligned}
\left\langle\Delta J^{2}\right\rangle & =\frac{\left\langle\Delta J^{2}(N)\right\rangle}{N} \\
& \approx \frac{N_{p}^{2} r_{p}^{2} \Delta x^{2}}{8 \gamma^{2} \sigma_{r}^{2}} \sum_{k=0}^{\infty}(2 k+1)^{2} G_{k}(a)^{2},
\end{aligned}
$$

where $a=J /(2 \varepsilon)$ and $G_{k}$ is defined in Eq. (B13). The derivation of Eq. (30) is explained in Appendix B and in Ref. [34]. The two types of noise cause a diffusion of the particle motion and result in emittance growth.

For the fluctuation of Eq. (24) the diffusion of $J$ per revolution is given by 


$$
\begin{aligned}
\left\langle\Delta J^{2}\right\rangle \approx & \frac{N_{p}^{2} r_{p}^{2} \Delta x^{2}}{8 \gamma^{2} \sigma_{r}^{2}} \\
& \times \sum_{n=-\infty}^{\infty} \sum_{k=0}^{\infty}(2 k+1)^{2} G_{k}^{2} \cos \left[(2 k+1) n \mu_{o}\right] e^{-|n| / \tau} \\
\approx & \frac{N_{p}^{2} r_{p}^{2} \Delta x^{2}}{8 \gamma^{2} \sigma_{r}^{2}} \sum_{k=0}^{\infty} \frac{(2 k+1)^{2} G_{k}(a)^{2} \sinh 1 / \tau}{\cosh 1 / \tau-\cos (2 k+1) \mu_{o}}
\end{aligned}
$$

Using the correlation of Eq. (29) the diffusion of $J$ for the second type of noise, Eq. (28), becomes

$$
\begin{aligned}
\left\langle\Delta J^{2}\right\rangle \approx & \frac{N_{p}^{2} r_{p}^{2} \Delta x^{2}}{16 \gamma^{2} \sigma_{r}^{2}} \sum_{k=0}^{\infty}(2 k+1)^{2} G_{k}(a)^{2} \sinh 1 / \tau \\
& \times\left[\frac{1}{\cosh 1 / \tau-\cos (2 k \mu-\delta \mu)}\right. \\
& \left.+\frac{1}{\cosh 1 / \tau-\cos (2(k+1) \mu+\delta \mu)}\right],
\end{aligned}
$$

where $\delta \mu$ is the tune difference between the weak and strong beam oscillations $\left(\delta \mu=\mu-\mu_{o}\right)$.

Figure 17 shows the diffusion rate of $J$ as a function of $J$, where $\nu=0.31$. The diffusion rate is proportional to the square of the fluctuation amplitude $\Delta x$ and to the square of the beam-beam parameter $\left(\propto N_{p}\right)$. The rate is normalized by the combined factor, $A=\left[N_{p} r_{p} \Delta x /\left(\gamma \sigma_{r}\right)\right]^{2} / 8$ in the figure. The diffusion rate depends on the tune except for turn-by-turn noise. For large $\tau$ and $\nu=m /(n+1)$, with integers $m$ and $n$, a small denominator for $(n=k)$ yields a high diffusion rate. The dependence on the tune is less strong for other tune values.

The rms emittance $\varepsilon$ equals the average value of the action $J$. Considering a Gaussian beam distribution the initial distribution function in action-angle variables is $\rho(J ; 0)=\exp (-J / \varepsilon) / 2 \pi \varepsilon)$.

The emittance growth can be obtained from the calculated diffusion rate. For a Hamiltonian system the Fokker-Planck equation describing the general time

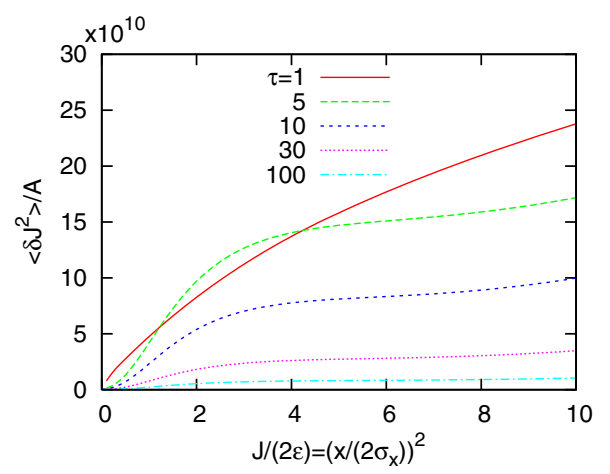

FIG. 17. Diffusion rate given by Eq. (31) for $\nu=0.31$. The rate is normalized by $A=\left(N_{p} r_{p} \Delta x / \gamma \sigma_{r}\right)^{2} / 8$. evolution of the phase-space distribution $\rho$ reduces to a diffusion equation [35]

$$
\frac{\partial \rho(J ; n)}{\partial n}=\frac{1}{2} \frac{\partial}{\partial J}\left(D(J) \frac{\partial \rho(J ; n)}{\partial J}\right)
$$

where $n$ is the turn number and $D(J) \equiv\left\langle\Delta J^{2}\right\rangle$ the diffusion coefficient.

The description is further simplified if the diffusion rate $\left\langle\Delta J^{2}\right\rangle$ is proportional to $J$, that is if $\left\langle\Delta J^{2}\right\rangle=D^{\prime} J$, in which case [34]

$$
\frac{\partial \rho(J ; n)}{\partial n}=\frac{1}{2} \frac{\partial}{\partial J}\left(D^{\prime} J \frac{\partial \rho(J ; n)}{\partial J}\right)
$$

The solution of this equation for an initial emittance $\varepsilon$ is given by

$$
\rho(J ; n)=\frac{1}{2 \pi\left(\varepsilon+D^{\prime} n / 2\right)} \exp \left(-\frac{J}{\varepsilon+D^{\prime} n / 2}\right) .
$$

In other words, for the linear diffusion $\left\langle\Delta J^{2}\right\rangle=D^{\prime} J$, the emittance increases by $\Delta \varepsilon=D^{\prime} / 2$ every turn. The emittance growth rate per turn can be expressed as

$$
\frac{\Delta \varepsilon}{\varepsilon}=\frac{D^{\prime}}{2 \varepsilon}=\frac{1}{4 \varepsilon^{2}} \frac{d\left\langle\Delta J^{2}\right\rangle}{d a},
$$

where $a=J /(2 \varepsilon)$.

Figure 17 shows that indeed the diffusion rate is approximately proportional to $J$ for small values of $J /(2 \varepsilon)<2$, or $x / \sigma_{x}<2.82$. Fitting the slope of $\left\langle\Delta J^{2}\right\rangle$ for turn-by-turn noise $(\tau=1)$ gives

$$
\frac{d\left\langle\Delta J^{2}\right\rangle}{d a} \approx \frac{N_{p}^{2} r_{p}^{2}}{8 \gamma^{2}} \frac{\Delta x^{2}}{\sigma_{r}^{2}} \times 4.4 .
$$

The luminosity degradation rate per collision is then estimated from the emittance growth rate as

$$
\frac{\Delta L}{L} \approx-\left(\xi \frac{\Delta x}{\sigma_{r}}\right)^{2} \times 21.7 .
$$

For multi-IPs with the same collision condition, the same noise level and superperiodicity of tune, the formula is modified by

$$
\frac{\Delta L}{L}=-\left(\xi_{\mathrm{tot}} \frac{\Delta x}{\sigma_{r}}\right)^{2} \times \frac{21.7}{N_{\mathrm{IP}}},
$$

where $N_{\text {IP }}$ is the number of IPs.

Under the same conditions the tolerance on the noise amplitude is obtained from the one-day luminosity lifetime $\Delta L / L=10^{-9}$ for two IPs $\left(N_{\text {IP }}=2\right)$ as 


$$
\xi_{\text {tot }} \frac{\Delta x}{\sigma_{r}} \approx 10^{-5}
$$

This equation was derived by linearly extrapolating the diffusion coefficient to larger amplitudes. As shown in Fig. 17 this extrapolation overestimates the actual diffusion coefficient at amplitudes above about 2 sigma, and, hence, it yields an upper bound on the luminosity decay.

Figure 18 shows the diffusion rates for the second type of noise, given by Eq. (28). Plots (a) and (b) refer to beamorbit oscillations with the same tune $(\delta \mu=0)$ and with a difference of $\delta \mu=\xi=0.01$, as could be due to the beambeam tune shift, respectively. In the top picture, for large correlation times a strong enhancement of the diffusion at small amplitudes is seen. The strong modulation at a frequency equal to the tune of the weak-beam particles drives an externally forced oscillation on resonance.

The diffusion rate for $\delta \mu=\xi$ in plot (b) may be a better representation of the real beam-beam system. For $\tau>1$ this diffusion rate saturates at $J /(2 \varepsilon) \approx 1$, and it assumes values similar to the one for $\tau=1$ below $3.5 \sigma$, or for $J /(2 \varepsilon)<3$.

The analytic theory considers a near solvable system, normally far from resonances. There is no such limitation in the beam-beam simulations. The only drawback is that considerable computing time is required to evaluate a slow emittance growth by means of simulations. Simulating the external noise is straightforward. Namely, a modulation is applied to the strong beam according to Eq. (24) or (28).
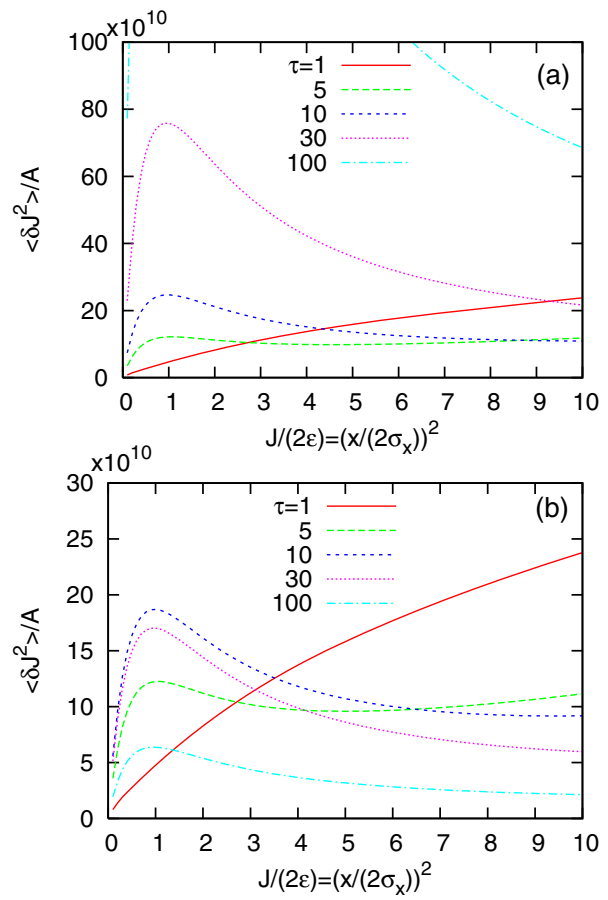

FIG. 18. Diffusion rate given by Eq. (32) as a function of $J /(2 \epsilon)$ for equal tunes $\delta \mu=0$ (top) or with a tune difference of $\delta \mu=\xi=0.01$ (bottom). The rate on the vertical axis is normalized by the factor $A=\left[N_{p} r_{p} \Delta x /\left(\gamma \sigma_{r}\right)\right]^{2} / 8$.
The effects of resonances, longitudinal motion, and crossing angle are easily taken into account in the simulations.

We first discuss weak-strong simulations including an external noise with $\tau=1$. Figure 19 shows the simulated luminosity degradation for collisions without crossing angle. The degradation is plotted as a function of the fluctuation amplitude for three values of the total beambeam parameter, $\xi_{\text {tot }}=0.02,0.04$, and 0.05 . The corresponding three predictions from the analytic formula Eq. (40) are also drawn in the figure. The simulation results and the formula agree fairly well.

If the noise contains a tune frequency component, the weak-strong model, in which the noise is treated as an external driving force, leads to ambiguous results, since the noise induces a coherent beam-beam mode. The luminosity degradation due to various types of noise has also been studied through strong-strong simulations $[3,36]$. Here the limited number of macroparticles induces numerical noise, e.g., $\delta x / \sigma_{x}=0.001$ for 1 million macroparticles, and the simulation time is much longer than the one needed for the weak-strong simulations. Figure 20 shows the simulated luminosity decrement as a function of the noise amplitude $\Delta x$. The strong-strong simulation is performed in $10^{6}$ turns with the macroparticle number $10^{6}$. Luminosity decrement is estimated as $5.3 \times 10^{-9}$ using Eq. (38) $\left(\xi_{\mathrm{IP}}=0.011\right)$ for only the statistical noise $\delta x / \sigma_{x}=0.001$ of the strongstrong simulation. The luminosity decrement depends on the value of $\Delta x$, but it is insensitive to $\delta x$ for $\tau>1$. The line from Eq. (39) is also shown in Fig. 20. At small noise amplitude $\left(\Delta L / L_{0}<20 \times 10^{-9}\right)$, i.e., the amplitudes of practical interest, the simulated luminosity decrement is close to the analytical formula. Shown in the figure are the luminosity decrements computed over several correlation times. The decrement scales with $\Delta x$, independently of the value of $\tau$. This behavior could be related to the saturation of the diffusion rate in Fig. 18(b). In the future, increasing computer power will enable more detailed strong-strong beam-beam simulations which may yield an even better

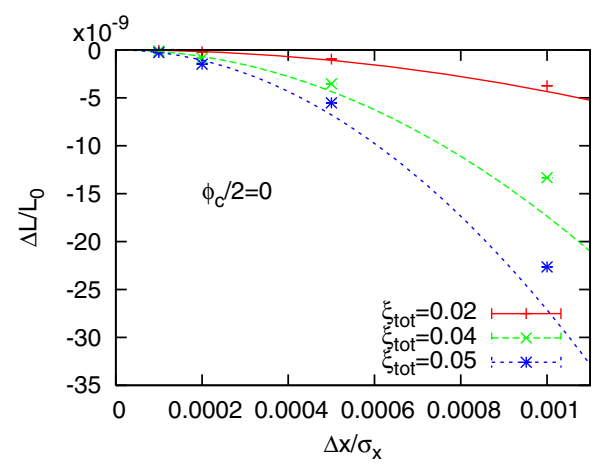

FIG. 19. Luminosity decrement as a function of the offset noise amplitude (for $\tau=1$ ) in collisions without a crossing angle obtained from a weak-strong simulation (plotting symbols) compared with the predictions from Eq. (38) (the lines), for the working point $\left(\nu_{x}, \nu_{y}\right)=(0.31,0.32)$. 


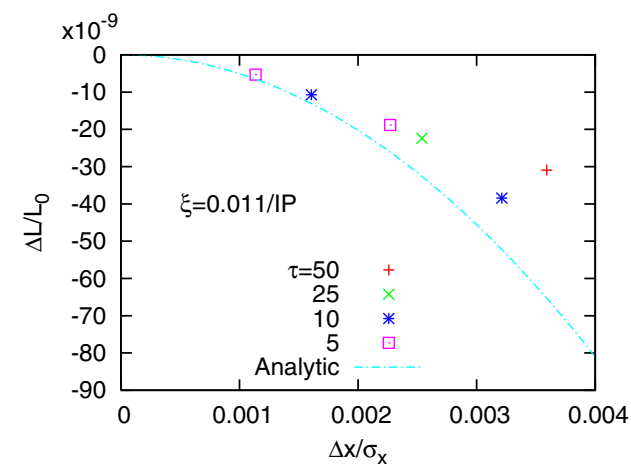

FIG. 20. Luminosity decrement as a function of the offset-noise amplitude. Results from a 2-dimensional strong-strong simulation (plotting symbols) are compared with predictions from Eq. (38), for the working point $\left(\nu_{x}, \nu_{y}\right)=(0.31,0.32)$. The simulation is performed in $10^{6}$ turns with the macroparticle number $10^{6}$.

understanding of the beam-beam behavior in the presence of noise.

Now we consider the luminosity performance in the presence of both a crossing angle and external noise. In Fig. 21 the luminosity degradation is shown for collisions with a crossing angle $\left(\theta_{c}=290 \mu \mathrm{rad}\right)$, corresponding to a Piwinski angle of $\theta_{c} \sigma_{z} /\left(2 \sigma_{r}\right)=0.89$. The beam-beam tune shift with this crossing angle is $75 \%$ of that for a head-on collision. The simulations were executed for the same bunch intensity as those without a crossing angle in Fig. 19. Predictions from Eq. (38) are also drawn in the figure. The simulated decrements are smaller than those expected from the analytical formula, but the difference appears unrelated to the presence of a nonzero crossing angle (compare Fig. 19).

Figure 22 shows the luminosity degradation as a function of the beam-beam parameter with two offset noises of amplitude $\Delta x / \sigma_{x}=0.0002$ and 0.001 , respectively. Predictions from Eq. (38) are also displayed in the

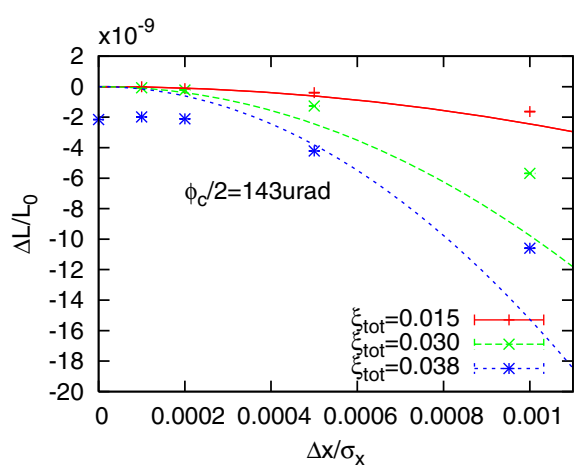

FIG. 21. Luminosity decrement as a function of the offsetnoise amplitude for collision with a nonzero crossing angle. Results from a weak-strong simulation (plotting symbols) are compared with predictions from Eq. (38), for the working point $\left(\nu_{x}, \nu_{y}\right)=(0.31,0.32)$.
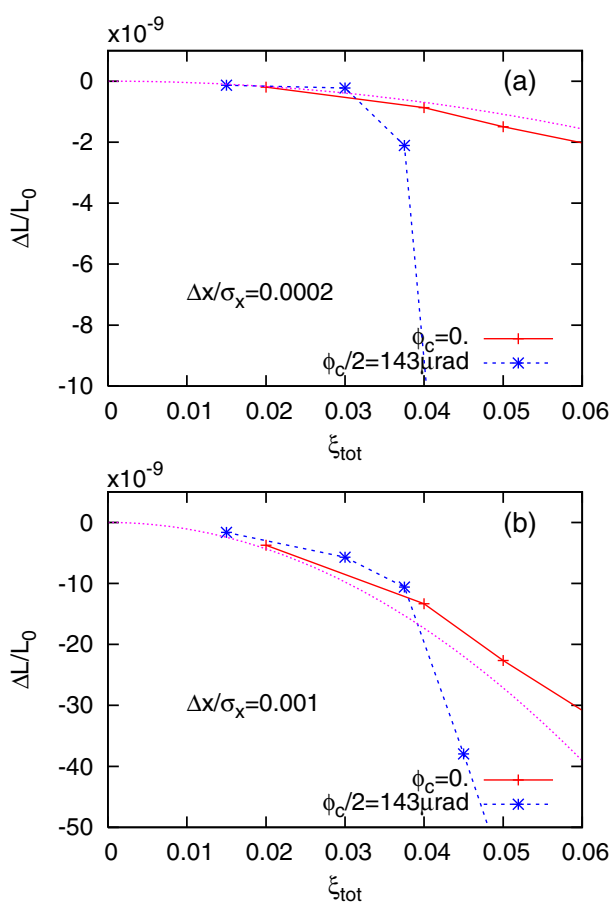

FIG. 22. Luminosity degradation as a function of the beambeam parameter under an offset noise of two different amplitudes, with and without a crossing angle.

figure. The tune shift is $25 \%$ lower for the crossing-angle collision. The luminosity degradation due to noise is fairly independent of the crossing angle. At higher beam-beam parameter $\xi_{\text {tot }}>0.038$, the luminosity degradation due to the crossing angle becomes dominant.

As far as the response to the external noise is concerned, there is no qualitative difference for the collisions with or without crossing angle. The degradation due to the crossing angle experienced for $\xi_{\text {tot }}=0.038$ is observed even without any noise, while a clear cross-talk between the effects of the crossing angle and of the noise is not seen. The degradation of the luminosity due to the fluctuation depends on $\xi_{\text {tot }}$, but it only weakly depends on the presence of the crossing angle. Basically emittance growth should be enhanced by noise near resonances, especially near low-order resonances. Seventh order resonances, which appear in crossing or offset collision, are weak in solely. The emittance growth due to a crossing angle is driven by the variation of transverse resonances via the synchrotron motion. On the other hand, the effect of noise, especially of turn-byturn noise, is independent of the tune in the tune area far from strong resonances, and it is insensitive to the presence of such weak resonances as is evident in the above simulation results and analytical treatments.

\section{CONCLUSIONS}

At hadron colliders the beam-beam limit manifests itself in the form of luminosity degradation and enhanced 
emittance growth. In this paper the beam-beam limit in the LHC has been studied in view of several possible limiting mechanisms, focusing on collisions between two bunches.

We have found that there is no fundamental limitation up to total tune shifts of at least 0.2 , in case of a perfect round beam collision at the nominal fractional tunes of the LHC, $\left(\nu_{x}, \nu_{y}\right)=(0.31,0.32)$.

Our results indicate a hurdle $\xi_{\text {tot }} \approx 0.038-0.05$ in the presence of a transverse offset or nonzero crossing angle. The crossing angle and the offset collision both induce additional transverse resonances due to the breaking of the symmetry $(x \rightarrow-x, y \rightarrow-y)$. By contrast, the diffusion caused by the purely transverse resonances alone appears weak.

For collisions with a nonzero crossing angle, the beambeam force depends on $z$. The synchrotron motion together with such a $z$-dependent beam-beam force greatly enhances the diffusion. The beam-beam limit with a finite crossing angle (for the nominal LHC Piwinski angle $\phi_{\text {piw }} \approx 0.89$ ) and including synchrotron motion is around 0.038 for the luminosity decrement $10^{-9}$ /turn ( 1 day). This limit depends on the operating point in tune space, because of the amplitude-dependent tune shift and the varying distance from resonances. At certain operating points, the beam-beam tune shift can also be limited by a coherent instability.

The beam-beam limit due to optical errors may be a more practical concern. IP dispersion results in a collision offset which varies with the energy deviation, e.g., with the phase of the synchrotron motion. Resonances excited by the offset are modulated by the synchrotron motion, enhancing the emittance growth. Nonzero chromaticity and chromatic beta induce an additional tune modulation at the synchrotron frequency, which aggravates the effect of the resonances driven by crossing angle or collision offset. A nonzero $x-y$ coupling at the interaction point also affects the luminosity performance. Nonzero coupling parameters $r_{1}, r_{2}$, and $r_{4}$ break the symmetry of the collision and induce new resonances. The luminosity degradation is further enhanced by a crossing angle or by nonzero chromaticity, for the same reason as in the case of dispersion. For hadron, the tolerances with respect to residual IP coupling are relaxed compared with those at (flat-beam) electron-positron colliders, e.g., at KEKB.

We did not consider any realistic ring lattices in this paper. The essential questions for judging the importance of the lattice are whether the resonances induced by the nonlinear magnetic fields lie within the tune footprint area of the beam-beam force, whether their resonance widths $(\Delta J)$ are sufficiently large, and if these widths and the resonant amplitudes $\left(J_{R}\right)$, vary as a result of synchrotron motion. In general, the effect of the resonances is more serious in cases with a nonzero crossing angle and/or nonzero chromaticity.
Fast fluctuations of the collision offset are a serious issue for proton colliders. For the (HL-)LHC the use of crab cavities is foreseen in order to recover the geometrical luminosity loss otherwise induced by a large crossing angle. Any rf phase noise of these crab cavities will result in a transverse collision offset. In this article the dependence of the noise tolerance on the beam-beam parameter was evaluated by weak-strong and strong-strong model with colliding beam fluctuations. The result is described by a simple formula [Eq. (40)]. As more detailed strong-strong simulations are being performed with increasing computer power, an even better understanding of the noise behavior may be achieved in the not-too-distant future.

\section{ACKNOWLEDGMENTS}

The authors would like to thank Y. Alexahin, W. Herr, J. Qiang, S. Paret, T. Pieloni, T. Sen, R. Tomas, A. Valishev, and D. Zhou for helpful discussions. In particular, the formulas in Appendix B are based on discussions with $\mathrm{T}$. Sen [37]. This work was supported in part by the European Commission under the FP7 Research Infrastructures project EuCARD, Grant Agreement No. 227579, under the Capacities 7th Framework Programme project EuCARD-2, Grant Agreement No. 312453, and under the HORIZON 2020 Framework Programme project EuroCirCol, Grant Agreement No. 654305. This work is also supported by the Large Scale Simulation Program of High Energy Accelerator Research Organization (KEK).

\section{APPENDIX A: HAMILTONIAN COEFFICIENTS}

A code named SAD+ [38] based on differential algebra [39] calculates the one-turn transfer map $\boldsymbol{x}=\boldsymbol{f}\left(\boldsymbol{x}_{0}\right)$ containing the beam-beam interaction of Eq. (4) and linear transformation including crossing angle are expanded into a 12 th order Taylor series. In the code, $H$, which generates the nonlinear transfer map, is obtained by a polynomial integration,

$$
H=1-\int S_{i j} R^{-1} f_{j}(\boldsymbol{x}) d x_{i},
$$

where $R$ is the linear map of $\boldsymbol{f}$, and $S_{i j}$ the symplectic metric.

In the following we consider one LHC interaction point with horizontal crossing.

The variables $(\boldsymbol{x})$ in $H$ are converted to the action variables $\left(J_{x}, \phi_{x}, J_{y}, \phi_{y}\right) . H$ is represented by Fourier components for the betatron phase $\phi$ in Eq. (14). $H_{00}$, which is independent of $\phi$, then gives the amplitudedependent tune shift. Considering $H$ and $J$ with the dimension of meter the expansion in action variables read 


$$
\begin{aligned}
H_{00}\left(J_{x}, J_{y}\right)= & 2.01 \times 10^{42} J_{x}^{6}+1.21 \times 10^{43} J_{x}^{5} J_{y}-4.82 \times 10^{33} J_{x}^{5}+2.80 \times 10^{43} J_{x}^{4} J_{y}^{2}-2.27 \times 10^{34} J_{x}^{4} J_{y} \\
& +1.14 \times 10^{25} J_{x}^{4}+1.30 \times 10^{44} J_{x}^{3} J_{y}^{3}-7.27 \times 10^{34} J_{x}^{3} J_{y}^{2}+4.64 \times 10^{25} J_{x}^{3} J_{y}-2.71 \times 10^{16} J_{x}^{3} \\
& +1.67 \times 10^{44} J_{x}^{2} J_{y}^{4}-1.49 \times 10^{35} J_{x}^{2} J_{y}^{3}+1.19 \times 10^{26} J_{x}^{2} J_{y}^{2}-8.66 \times 10^{16} J_{x}^{2} J_{y}+6.10 \times 10^{7} J_{x}^{2} \\
& +1.79 \times 10^{44} J_{x} J_{y}^{5}-1.72 \times 10^{35} J_{x} J_{y}^{4}+1.69 \times 10^{26} J_{x} J_{y}^{3}-1.58 \times 10^{17} J_{x} J_{y}^{2}+1.36 \times 10^{8} J_{x} J_{y} \\
& +1.07 \times 10^{44} J_{y}^{6}-1.21 \times 10^{35} J_{y}^{5}+1.40 \times 10^{26} J_{y}^{4}-1.60 \times 10^{17} J_{y}^{3}+1.74 \times 10^{8} J_{y}^{2} .
\end{aligned}
$$

For simplicity we have dropped the explicit appearance of units. Coefficients which are multiplied by the $n$th power of action variables should have the unit $\mathrm{m}^{-n+1}$.

At certain amplitudes a resonance condition is satisfied, as is shown in Eq. (15). This paper focuses on seventhorder resonances. Four lines corresponding to the resonances, $\left(m_{x}, m_{y}\right)=(7,0),(5,2),(3,4)$, and $(1,6)$ are excited by a horizontal crossing. Fourier components of odd order for $m_{y}$ do not appear due to the $\pm y$ symmetry of the horizontal crossing. [A vertical crossing would drive other seventh-order resonances, namely $\left(m_{x}, m_{y}\right)=(6,1),(4,3)$, $(2,5)$, and $(0,7)$.] The Fourier components related to the seventh-order resonances are given by the terms $G_{70}, G_{52}$, $G_{34}$, and $G_{16}$ with similar polynomials.

Using Eq. (A2), lines in amplitude space satisfying the resonance conditions of Eq. (15) can be calculated. The resonance width is evaluated using Eq. (16) by substituting the amplitudes $J_{x}=J_{x, R}$ and $J_{y}=J_{y, R}$ in $G_{m_{x} m_{y}}$.

\section{APPENDIX B: EMITTANCE GROWTH DUE TO COLLISION OFFSET NOISE IN THE WEAK-STRONG MODEL}

This appendix is based on $[34,37]$. The beam-beam force (potential) with the bunch population $\left(N_{p}\right)$ and the transverse size $\left(\sigma_{r}\right)$ is expressed by

$$
U(x)=\frac{N_{p} r_{p}}{\gamma_{p}} \int_{0}^{\infty} \frac{1-e^{-x^{2} /\left(2 \sigma_{r}^{2}+q\right)}}{2 \sigma_{r}^{2}+q} d q
$$

where $r_{p}$ and $\gamma_{p}$ are the classical radius of the proton and the relativistic factor of the (weak) beam, respectively. The betatron variables are represented by the normalized action variable $(a)$ and phase $(\psi)$ as follows:

$$
\begin{gathered}
x=\sqrt{2 \beta^{*} J} \cos \psi=2 \sigma_{r} \sqrt{a} \cos \psi, \\
\beta^{*} p=-\sqrt{2 \beta^{*} J} \sin \psi=-2 \sigma_{r} \sqrt{a} \sin \psi,
\end{gathered}
$$

where

$$
a=\frac{J}{2 \varepsilon}=\frac{\beta^{*} J}{2 \sigma_{r}^{2}}=\frac{x^{2}+\beta^{* 2} p^{2}}{4 \sigma_{r}^{2}} .
$$

The potential is expanded by Fourier series of the betatron phase as follows:

$$
U(x)=\frac{N_{p} r_{P}}{\gamma_{p}} \sum_{k=0}^{\infty} U_{k}(a) \cos 2 k \psi .
$$

The Fourier component is expressed by

$U_{k}(a)=\int_{0}^{a}\left[\delta_{0 k}-\left(2-\delta_{0 k}\right)(-1)^{k} e^{-w} I_{k}(w)\right] \frac{d w}{w}$,

where $I_{k}$ is the modified Bessel function. The change of $J$ per one revolution is given by the derivative of the beambeam potential for $\psi$ as follows:

$$
\Delta J=-\frac{\partial U}{\partial \psi}=\frac{N r_{p}}{\gamma} \sum_{k=0}^{\infty} 2 k U_{k} \sin 2 k \psi
$$

This change, which indicates a stable sinusoidal modulation of the betatron amplitude, does not induce emittance growth.

We consider the case in which the strong beam has a small offset $(\Delta x)$, The beam-beam potential with the offset is expanded for $\Delta x$,

$$
U(x+\Delta x)=U(x)+U^{\prime}(x) \Delta x .
$$

$\Delta x$ is a random variable fluctuated by Eq. (24) or (28).

Derivative of the beam-beam potential for the offset $(\Delta x)$ is represented by $(a, \psi)$,

$$
U^{\prime}(a, \psi)=\frac{\partial U}{\partial a} \frac{\partial a}{\partial x}+\frac{\partial U}{\partial \psi} \frac{\partial \psi}{\partial x} .
$$

Using the relations

$$
\frac{\partial a}{\partial x}=\frac{\sqrt{a}}{\sigma_{r}} \cos \psi
$$

and

$$
\frac{\partial \psi}{\partial x}=-\frac{1}{2 \sigma_{r} \sqrt{a}} \sin \psi
$$

each term of Eq. (B8) is expressed as 


$$
\begin{aligned}
\frac{\partial U}{\partial a} \frac{\partial a}{\partial x} & =\frac{N_{p} r_{p}}{\gamma \sigma_{r}} \sum_{k=0}^{\infty} \sqrt{a} U_{k}^{\prime} \cos 2 k \psi \cos \psi \\
& =\frac{N_{p} r_{p}}{2 \gamma \sigma_{r}} \sum_{k=0}^{\infty} \sqrt{a}\left(U_{k+1}^{\prime}+U_{k}^{\prime}\right) \cos (2 k+1) \psi
\end{aligned}
$$

and

$$
\begin{aligned}
\frac{\partial U}{\partial \psi} \frac{\partial \psi}{\partial x} & =\frac{N_{p} r_{p}}{2 \gamma \sigma_{r} \sqrt{a}} \sum_{k=0}^{\infty} 2 k U_{k} \sin 2 k \psi \sin \psi \\
& =\frac{N_{p} r_{p}}{2 \gamma \sigma_{r} \sqrt{a}} \sum_{k=0}^{\infty}\left[(k+1) U_{k+1}-k U_{k}\right] \cos (2 k+1) \psi
\end{aligned}
$$

The Fourier expansion of the potential with respect to the offset now becomes

$$
U^{\prime}(J, \psi)=\frac{N_{p} r_{p}}{2 \gamma \sigma_{r}} \sum_{k=0}^{\infty} G_{k}(a) \cos (2 k+1) \psi,
$$

where

$G_{k}(a)=\sqrt{a}\left[U_{k+1}^{\prime}+U_{k}^{\prime}\right]+\frac{1}{\sqrt{a}}\left[(k+1) U_{k+1}-k U_{k}\right]$,

and $U_{k}^{\prime}$ is the derivative evaluated at $a=J /(2 \varepsilon)$.

The diffusion rate $\left\langle\Delta J^{2}(N)\right\rangle / N$ is estimated as

$$
\begin{aligned}
\left\langle\Delta J^{2}(N)\right\rangle= & \sum_{\ell=1}^{N} \sum_{n=-\ell+1}^{N-\ell} \frac{\partial U^{\prime}(\ell)}{\partial \psi} \frac{\partial U^{\prime}(\ell+n)}{\partial \psi}\left\langle\Delta x_{\ell} \Delta x_{\ell+n}\right\rangle \\
= & \frac{N_{p}^{2} r_{p}^{2} \sigma_{r}^{2}}{4 \gamma^{2}} \sum_{\ell=1}^{N} \sum_{n=-\ell}^{N-\ell} \sum_{k=0}^{\infty} \sum_{j=0}^{\infty}(2 k+1)(2 j+1) G_{k} G_{j} \sin \left[(2 k+1) \psi_{\ell}\right] \sin \left[(2 j+1) \psi_{\ell+n}\right]\left\langle\Delta x_{\ell} \Delta x_{\ell+n}\right\rangle \\
= & \frac{N_{p}^{2} r_{p}^{2}}{8 \gamma^{2} \sigma_{r}^{2}} \sum_{\ell=1}^{N} \sum_{n=-\ell}^{N-\ell} \sum_{k=0}^{\infty} \sum_{j=0}^{\infty}(2 k+1)(2 j+1) G_{k} G_{j}\left\{\cos \left[(2 k-2 j) \psi_{\ell}-(2 j+1) 2 \pi n \mu\right]\right. \\
& \left.-\cos ^{2}\left[(2 k+2 j+2) \psi_{\ell}+(2 j+1) 2 \pi n \nu\right]\right\}\left\langle\Delta x_{\ell} \Delta x_{\ell+n}\right\rangle \\
\approx & \frac{N_{p}^{2} r_{p}^{2}}{8 \gamma^{2} \sigma_{r}^{2}} N \sum_{n=-\infty}^{\infty} \sum_{k=0}^{\infty}(2 k+1)^{2} G_{k}^{2} \cos [2 \pi(2 k+1) n \nu] \Delta x^{2} e^{-|n| / \tau} \\
= & \frac{N_{p}^{2} r_{p}^{2}}{8 \gamma^{2}} \frac{\Delta x^{2}}{\sigma_{r}^{2}} N \sum_{k=0}^{\infty} \frac{(2 k+1)^{2} G_{k}(a)^{2} \sinh 1 / \tau}{\cosh 1 / \tau-\cos (2 k+1) \mu_{o}}
\end{aligned}
$$

where $\psi_{\ell+n}=\psi_{\ell}+2 \pi \nu$ and, in the last step, we have used the formula

$$
\sum_{n=-\infty}^{\infty} \cos [2 \pi(2 k+1) n \nu] e^{-|n| / \tau}=\frac{\sinh 1 / \tau}{\cosh 1 / \tau-\cos (2 k+1) \mu_{o}}
$$

Only the $O(N)$ term for $k=j$ has been kept in the second to last line of Eq. (B14). Dividing Eq. (B14) by $N$ yields Eq. (31).

For the second type of noise, Eq. (32) is obtained by replacing Eq. (26) with Eq. (29) in Eq. (B14).

[1] R. Assmann and K. Cornelis, in Proceedings of the 7th European Particle Accelerator Conference, Vienna, Austria, 2000 (EPS, Geneva, 2000), p. 1187.

[2] J. Gareyte, in Proceedings of the Workshop on Beam-Beam Effects in Large Hadron Colliders (LHC'99), Geneva,
1999, edited by F. Zimmermann and J. Poole (CERN, 1999); F. Zimmermann and J. PooleReport No. CERN-SL99-039-AP.

[3] K. Ohmi et al., in Proceedings of the 22nd Particle Accelerator Conference, PAC-2007, Albuquerque, NM (IEEE, New York, 2007), p. 1496, TUPAN048; K. Ohmi et al., Report No. CERN-LHC-PROJECT-REPORT-1048.

[4] W. Herr et al., in Proceedings of the 2nd International Particle Accelerator Conference, IPAC-2011, San Sebastián, Spain (EPS-AG, Spain, 2011), p. 1936.

[5] W. Herr et al., in Proceedings of LHC Performance Workshop on Chamonix, France, 2012, Report No. CERN-2012-006, 2012, p. 99.

[6] E. G. Stern, J. F. Amundson, P. G. Spentzouris, and A. A. Valishev, Phys. Rev. ST Accel. Beams 13, 024401 (2010).

[7] W. Fischer et al., ICFA Beam Dynamics Newsletter 52, 102 (2010).

[8] Y. Luo, W. Fischer, N. P. Abreu, X. Gu, A. Pikin, and G. Robert-Demolaize, Phys. Rev. ST Accel. Beams 15, 051004 (2012).

[9] V. V. Danilov, P. M. Ivanov, E. A. Perevedentsev, E. A. Simonov, and A. N. Skrinsky, in Proceedings of 
the Workshop on Beam-Beam and Beam Radiation Interactions, Los Angeles, CA, 1991 (World Scientific, Hackensack, NJ, 1991), Vol. 1, p. 1.

[10] K. Hirata et al., Particle Accelerators 40, 205 (1993).

[11] K. Oide and K. Yokoya, Phys. Rev. A 40, 315 (1989).

[12] K. Hirata, Phys. Rev. Lett. 74, 2228 (1995).

[13] K. Ohmi, M. Tawada, Y. Cai, S. Kamada, K. Oide, and J. Qiang, Phys. Rev. ST Accel. Beams 7, 104401 (2004).

[14] Y. Zhang, K. Ohmi, and L. Chen, Phys. Rev. ST Accel. Beams 8, 074402 (2005).

[15] LHC design report, Report No. CERN-2004-003-V-1.

[16] V. V. Danilov and V. D. Shiltsev, Report No. FERMILABFN-0655, 1997.

[17] V. V. Danilov and E. A. Perevedentsev, in Proceedings of Advanced ICFA Beam Dynamics Workshop on Beam Dynamics Issues for e+e-Factories, Frascati, 321 (1997).

[18] K. Ohmi and K. Oide, Phys. Rev. ST Accel. Beams 10, 014401 (2007).

[19] J. Laskar, Icarus 88, 266 (1990).

[20] L. Nadolski and J. Laskar, Phys. Rev. ST Accel. Beams 6, 114801 (2003).

[21] D. Shatilov, E. Levichev, E. Simonov, and M. Zobov, Phys. Rev. ST Accel. Beams 14, 014001 (2011).

[22] A. Dragt and J. Finn, J. Math. Phys. (N.Y.) 17, 2215 (1976).

[23] G. Franchetti and F. Zimmermann, Phys. Rev. Lett. 109, 234102 (2012).

[24] K. Ohmi, Phys. Rev. E 62, 7287 (2000).

[25] A. W. Chao, K.-H. Mess, M. Tigner, and F. Zimmermann, Handbook of Accelerator Physics and Engineering, 2nd ed. (World Scientific, New Jersey, 2013).
[26] Y. Alexahin, Nucl. Instrum. Methods Phys. Res., Sect. A 480, 253 (2002).

[27] D. Zhou, K. Ohmi, Y. Seimiya, Y. Ohnishi, A. Morita, and H. Koiso, Phys. Rev. ST Accel. Beams 13, 021001 (2010).

[28] Y. Ohnishi, K. Ohmi, H. Koiso, M. Masuzawa, A. Morita, K. Mori, K. Oide, Y. Seimiya, and D. Zhou, Phys. Rev. ST Accel. Beams 12, 091002 (2009).

[29] K. Ohmi, Proceedings of the 5th International Particle Accelerator Conference, IPAC-2014, Dresden, Germany (EPS-AG, Dresden, 2014), TUPRI022.

[30] Y. Seimiya, K. Ohmi, D. Zhou, J. W. Flanagan, and Y. Ohnishi, Prog. Theor. Phys. 127, 1099 (2012).

[31] K. Ohmi and H. Koiso, Proceedings of the International Particle Accelerator Conference, Kyoto, Japan, 2010 (ICR, Kyoto, 2010), p. 1548.

[32] R. Tomas (private communication).

[33] K. Ohmi et al., Proceedings of HB2012 (TH01B03), Beijing, China, 2012, http://www.jacow.org/.

[34] T. Sen and J. A. Ellison, Phys. Rev. Lett. 77, 1051 (1996).

[35] F. Zimmermann, Ph.D. thesis, University of Hamburg, Report No. DESY 93-059, Appendix F, 1993.

[36] S. Paret and J. Qiang, Proceedings of the 3rd International Particle Accelerator Conference, New Orleans, LA, 2012 (IEEE, Piscataway, NJ, 2012), p. 1374.

[37] T. Sen (private communication).

[38] SAD Home Page, http://acc-physics.kek.jp/SAD/.

[39] M. Berz, Nucl. Instrum. Methods Phys. Res., Sect. A 298, 426 (1990). 